Documents pour l'histoire des techniques

Nouvelle série

$17 \mid 1^{\text {er }}$ semestre 2009

L'invention technique et les figures de l'inventeur (XVIII $-X^{\mathrm{e}}$ siècles)

\title{
Pollution industrielle et réglementation des manufactures et ateliers en France au XIXe siècle : les textes fondateurs
}

Industrial pollution and regulations for factories and workshops in France in the $X I X^{\text {th }}$ century : the first measures

Sabine Barles, André Guillerme et Laurence Lestel

\section{(2) OpenEdition}

Journals

Édition électronique

URL : http://journals.openedition.org/dht/363

DOI : $10.4000 /$ dht.363

ISSN : 1775-4194

Éditeur :

Centre d'histoire des techniques et de l'environnement du Cnam (CDHTE-Cnam), Société des élèves du CDHTE-Cnam

\section{Édition imprimée}

Date de publication : 31 mars 2009

Pagination : 174-218

ISBN : 978-2-95-30779-3-3

ISSN : 0417-8726

\section{Référence électronique}

Sabine Barles, André Guillerme et Laurence Lestel, « Pollution industrielle et réglementation des manufactures et ateliers en France au XIXe siècle : les textes fondateurs », Documents pour I'histoire des techniques [En ligne], $17 \mid 1^{\mathrm{er}}$ semestre 2009, mis en ligne le 01 octobre 2010, consulté le 07 septembre 2020. URL : http://journals.openedition.org/dht/363; DOI : https://doi.org/10.4000/dht. 363 


\title{
Pollution industrielle ef réglementation des manufactures et ateliers en France au XIX ${ }^{e}$ siècle : les textes fondateurs
}

\author{
Sabine Barles \\ Université Paris-VIII \\ André Guillerme \\ CDHTE-Cnam \\ Laurence Lestel \\ Université Paris-VI
}

\begin{abstract}
Résumé
Les textes réglementaires se rapportant aux nuisances sont peu connus. Ils révèlent une histoire complémentaire des techniques. En France, l'essor de l'industrialisation a généré des pollutions urbaines qui ont obligé les autorités politiques à prendre des mesures d'encadrement et de conseil à Paris, première ville industrielle à l'échelle nationale, au début du XIXe siècle, puis dans tout l'Empire par décret du 15 octobre 1810. Ces règlements déclinent les métiers dans leurs rapports au voisinage des fabriques.
\end{abstract}

Résumés et mots clés en anglais sont regroupés en fin de volume, accompagnés des mots clés français

\section{Préambule}

Des documents relatifs à l'histoire de la pollution Du fait même de sa formation initiale qui mêle histoire et géographie, tout historien aborde l'environnement pour cadrer son exposé ou décorer son propos, mais il ne s'y engage que rarement : l'environnement est une catégorie humaniste récente, la pollution plus encore'. Le champ de l'histoire des techniques

\footnotetext{
1 Au début des années 1980, quatre publications ouvrent, presque simultanément, le champ historiographique de l'environnement urbain : Martin V. Melosi éd., Pollution \& reform in American cities, 1870-1930, Austin, University of Texas Press, 1980 ; Alain Corbin, Le miasme et la jonquille, Paris, Aubier, 1982 ; Jean-Robert Pitte, Histoire du paysage en français, Paris, Tallandier, 1983 ; André Guillerme, Les temps de l'eau. La cité, l'eau et les techniques, Seyssel, Champ Vallon, 1983. Plus récemment, on peut mentionner, aux États-Unis, Christin M. Rosen et Joel A. Tarr éd., The environment and the city, Journal of urban history, $n^{\circ}$ spécial, 20, mai 1994; Joel A. Tarr, The search for the ultimate sink: urban pollution in historical perspective, Akron, The University of Akron Press, 1996. Pour la France, on citera Sabine Barles, La ville délétère: médecins et ingénieurs dans l'espace urbain XVIII -XIXe siècles, Seyssel, Champ Vallon, 1999 ; les habilitations à diriger les recherches de Laurence Lestel, « Tourner autour du plomb: de la batterie au lithium à la recherche
}

est pourtant rempli de métaux lourds, de produits toxiques, de matières en fermentation, de déchets recyclés engendrés par la production des arts et de l'industrie. Les techniques interviennent d'abord pour tirer parti de l'environnement et le modifier de façon plus ou moins irréversible. La pollution est un nouveau territoire de l'histoire technique. Ses vocables, ses analyses - chimique, biologique, mécanique — sont

des pollutions de notre passé industriel », Université d'Évry-Val d'Essonne, 2003 et de Geneviève Massard-Guilbaud, "Une histoire sociale de la pollution industrielle dans les villes françaises (1789-1914) II, Université Lyon-I, 2003 ; André Guillerme, Anne-Cécile Lefort, Gérard Jigaudon, Dangereux, insalubres, incommodes: paysages industriels en banlieve parisienne (XIX-XXe siècles), Seyssel, Champ Vallon, 2005 ; Estelle Baret-Bourgoin, La ville industrielle et ses poisons. Les mutations des sensibilités aux nuisances et pollutions industrielles à Grenoble, 1810-1914, Grenoble, Presses universitaires de Grenoble, 2005 ; Sabine Barles, L'invention des déchets urbains, France 1790-1970, Seyssel, Champ Vallon, 2005 ; Geneviève Massard-Guilbaud éd., Ville et environnement, dossier, Histoire urbaine, 18, 2007 ; André Guillerme, La naissance de I'industrie à Paris. Entre sueurs et vapeurs (1780-1830), Seyssel, Champ Vallon, 2007 ; André Guillerme, Historiographie de l'environnement urbain, Paris, PIRVE-CNRS, 2009. 
communs à l'histoire des techniques. L'agressivité de ces nuisances longtemps très urbaines mérite l'attention de l'historien du social, du travail, de l'économie et de l'écologie.

Bien que le terme de pollution ne soit employé dans son sens actuel que depuis un siècle et demi (un peu moins pour la France), les nuisances de toute nature engendrées par les activités humaines ont fait l'objet de nombreux écrits qui ont accompagné les révolutions industrielles et artisanales de la part de juristes, scientifiques, techniciens, édiles et citadins pour ne citer qu'eux. Ces textes nous permettent de contribuer non seulement à l'analyse de la perception de "ces révolutions industrielles qui influent si fort sur la population $\|^{2}$ par ceux qui les ont encouragées, accompagnées, vilipendées et ou subies et de tracer les contours d'une histoire sociale, économique, politique et technique de la pollution, mais aussi à la qualification voire à la quantification - avec les précautions qui s'imposent des nuisances engendrées par elles ; l'histoire nourrit ainsi la rétrospective, exercice nécessaire (mais pas suffisant) à la compréhension et à la gestion de l'environnement aujourd'hui et demain.

Les sources écrites de l'histoire de la pollution sont donc nombreuses si l'on considère les deux à trois derniers siècles : lois et règlements, rapports - des académies, des conseils de salubrité -, brochures à caractère plus ou moins pamphlétaire, articles dans les revues telles que les Histoire et Mémoires de l'Académie des sciences, les Histoire et Mémoires de la Société royale de médecine, les Annales d'hygiène publique et de médecine légale, les Annales des ponts et chaussées, les Annales de chimie et de physique, La technique sanitaire et municipale, etc. Bibliothèques, archives publiques et privées regorgent ainsi de témoignages et nous montrent - si nous voulons le voir - le long aveuglement de l'histoire économique, industrielle, technique relativement à la question des nuisances et des pollutions urbaines et industrielles.

Ce premier dossier est donc consacré aux textes réglementaires français et fondateurs en ce qui concerne les nuisances et pollutions engendrées par les manufactures et ateliers. D'autres suivront consacrés notamment à la Seine, à la rue et à l'espace public. Les règlements de police des métiers sont connus des médiévistes et des modernistes, mais ceux-ci restent pour l'essentiel municipaux. Le

2 Jacques Antoine Mourgue de Montredon, « Observations sur les naissances, les mariages \& les morts à Montpellier pendant dix années consécutives, de 1772 à 1781 inclusivement ॥, Histoire de la société royale de médecine, 1780-1781, p. 380. premier règlement national et sérieusement appliqué est le décret impérial du 15 octobre 1810 dont nous tentons de saisir la genèse parisienne, artisanale et industrielle, scientifique et académique.

\section{Pollution industrielle etréglementation des manufactures et ateliers}

En France, depuis les années 1770, l'artisanat évolve: des nouveaux métiers tentent de percer le bouclier corporatiste, de nouveaux procédés d'extraction ou de fabrication se développent tandis qu'émergent d'importantes manufactures de type industriel, marquées par le développement de la chimie et de la mécanique auquel la science est fortement associée. Ville pilote, la capitale paraît très diversifiée dans ses branches manufacturières : les fabriques les plus aérées côtoient les bouges.

À la Révolution, le tissu artisanal, encore lâche, est déjà disposé à Paris et dans ses campagnes. Derrière les barrières d'octroi des Fermiers généraux, on trouve douze mille ouvriers dans les filatures de coton - la principale est celle de Richard et Lenoir, rue de Charonne, qui occupe six établissements et près de quatre mille ouvriers - , trois mille pour le tulle et la gaze, un millier pour le papier peint. La Monnaie en occupe quinze cents. Entre ces pôles, une multitude de fabriques sous-traitantes tissent les liens, préparent les matériaux, recyclent les rebus pour faire de la colle, du carton, du verre, du laiton... Dans les premiers faubourgs, se dressent la manufacture de mécanique des frères Montgolfier à Chaillot où déjà souffre une grosse machine à vapeur chauffée à la houille, celle de Calla au faubourg Poissonnière, la filature de Lieven et Bauwens à Passy, la triperie de Robert, dans l'île aux Cygnes, qui nourrit le gras du Paris populaire, la manufacture de tabacs du Gros Caillou constituée en 1792 et qui emploie treize cents ouvriers en 1811, la tapisserie des Gobelins, le long de la Bièvre, et la grande miroiterie du Temple. À Montfaucon, la boyauderie produit des milliers de kilomètres de peau de chorizo, l'équarrissage abat une trentaine de chevaux par jour, la voirie fabrique la poudrette destinée à engraisser les terres maraîchères. Pour fabriquer le sel ammoniac, à Saint-Denis, Dizé distille vers 1788 des matières animales avec de l'acide chlorhydrique et du gypse dont la banlieve nord est la principale carrière ; sa fabrication arrêtée en 1790 est reprise par Pluvinet à Saint-Denis ${ }^{3}$, Payen et Bourlier à Vaugirard. Leurs établissements sont approvisionnés par les nombreux

3 Pluvinet est pendant la Révolution inspecteur commissaire des poudres pour la Normandie et le Nord. II est très lié à Lavoisier qu'il cherche à sauver de l'échafaud. 


\section{Documents relatifs à l'histoire de la pollution}

débris de matières animales de la capitale. À Sèvres se dresse la manufacture de porcelaine, à Jouy, celle des toiles peintes. Sur les hauteurs, Montmartre, Belleville, Issy, Montreuil pointent les moulins à vent pour broyer moins le froment que les graines oléagineuses destinées à la consommation, alors en forte croissance, de l'éclairage. Dans ces faubourgs, économistes et idéologues subodorent d'immenses richesses, des bras, des mains, des mécaniques, des fabriques.

Les guerres révolutionnaires, la levée en masse exigent la réquisition des savants restés fidèles à la République pour fournir le nécessaire aux armées. Jean-Antoine Chaptal, Lovis-Bernard GuytonMorveau et Gaspard Monge sont mobilisés pour fabriquer rapidement du salpêtre, notamment dans les faubourgs de la capitale - le sol calcaire y est propice - et réussissent dès 1792 à quintupler la production grâce aux nitrières artificielles ${ }^{4}$. Le procédé mis au point par Armand Seguin en 1794 pour tanner le cuir - la jusée - permet de le fabriquer en trois semaines au lieu d'une année : les 240000 peaux préparées annuellement dans sa manufacture de l'île de Sèvres couvrent alors un quart des besoins de l'armée révolutionnaire. Les scientifiques montrent ainsi qu'ils sont aussi techniciens et qu'ils sont utiles à la nation.

"Alors de grands établissements ont été formés sur toute la surface de la France ; on a perfectionné les opérations, on a simplifié les moyens $"{ }^{5}$ souligne Chaptal. L'industrie révolutionnaire et consulaire est d'abord mécanicienne parce que l'automate permet un énorme gain de productivité - notamment dans les filatures. À cette phase toujours bruyante par le cliquetis des mécanismes, il faut associer la fabrication en grand de molécules minérales-soude, acides - ou semi-organiques - sulfate, bleu de Prusse, alun - qui exige d'importants stocks d'eau claire ; une grande consommation de combustibles - bois, charbon de bois, houille - qui provoquent, par les fumées, une acidité corrosive des pluies et donc des eaux de ruissellement' .

Ces fabriques nouvelles, ces "start-up " qui

4 Chaptal est directeur de la poudrière de Grenelle en 1794. Cette année, il fait passer la production de 8000 à 35000 livres; 5 Jean-Antoine Chaptal, De l'industrie française, Paris, 1818, rééd. sous la dir. de Louis Bergeron, Paris, Imprimerie nationale, 1993, p. 285.

6 Liliane Hilaire-Pérez, "Le vol de déchets dans l'industrie en France et en Angleterre au XVIII siècle. Jalons pour une histoire comparée de l' 'embezzlement' "), dans Benoît Garnot éd., La petite délinquance du Moyen Age à l'époque contemporaine, Dijon, EUD, 1998, pp. 281-308. fleurissent dans et autour des grandes villes entre 1790 et 1800 s'installent généralement dans des bâtiments existants. Elles tirent parti de la crise foncière qui laisse à vil prix les édifices religieux - des forges s'y installent - et leurs dépendances - des moulins à eau. Elles absorbent des résidences secondaires aristocratiques qui comprennent le logis principal, pour le maître, des dépendances, pour les ateliers, des écuries et des réserves pour le stockage des matières premières et des récipients, l'accès à la voie publique, la clôture, l'isolement. Souvent plantées trop rapidement dans le paysage urbain, elles sont plus ou moins malodorantes. Elles dégagent des fumées, une odeur inconnue etjugée par conséquent dangereuse, voire toxique par le voisinage. Ainsi des fabriques d'acides : l'acide sulfurique étant obtenu par la combustion d'un mélange de soufre et de salpêtre, il se dégage comme une odeur d'allumette qu'on brûle, une odeur d'enfer qui laisse croire que le diable rôde dans son voisinage... " Les fabricants de soude se sont vus forcés de diriger les vapeurs acides dans des souterrains, ou de les perdre dans des puits profonds, pour ne pas brûler les récoltes, et incommoder les voisins, par ces nuages de vapeurs d'acide muriatique qui s'élèvent de leurs fourneaux $)^{7}$. On accuse donc ces fabricants de dégrader le sol et d'empoisonner les récoltes. La distillation des matières animales pour produire de l'ammoniaque dégage des odeurs fétides d'autant plus abondantes que la production s'amplifie : la fabrique de bleu de Prusse qui s'implante rue Saint-Nicolas, dans le Faubourg Saint-Martin, ligue les voisins et le maire, aisé et nommé par le préfet ${ }^{8}$. Parmi ceux qui contestent ces implants on trouve des propriétaires fonciers toujours plus puissants à mesure qu'on pénètre le cœur de la ville, toujours plus sensibles aux fluctuations de la rente foncière dont ils vivent, censitaires. On trouve aussi des artisans locataires riches et influents qui occupent depuis longtemps de grosses parcelles périphériques, des locataires qui sont employés par la fabrique et qui manifestent sourdement leur mécontentement en engendrant la rumeur. Le grand établissement industriel s'impose d'emblée en conquérant. La grande ville rentière, toujours sur la défensive, ne peut que réagir négativement.

Les outils politico-juridiques ne manquent pas pour réprimer les excès : en vertu de la loi du 24 avril 1790, les tribunaux sont compétents pour statuer sur tous les dommages causés à la propriété, donc pour juger de l'incommodité de certains métiers; la

7 J.-A. Chaptal, De l'industrie française, op. cit., note 4, p. 283. 8 Rapport du Conseil de salubrité n 18 du 15 février 1807. Archives de la Préfecture de Police (Paris), semi usuel. 
loi du 13 novembre 1791 maintient (provisoirement) les règlements de police relatifs à la création et à l'interdiction des manufactures dangereuses et en confie la gestion aux autorités municipales. Avant la Révolution, les Parlements étaient dans l'ensemble timorés vis-à-vis de l'industrie' et depuis la promulgation de cette loi, les édiles semblent faire preuve de beaucoup d'arbitraire, parfois d'hostilité, envers les nouveaux métiers. La puissance publique, très favorable à l'industrie, cherche à la développer en mettant en place des instruments promotionnels.

Pour assurer définitivement la liberté et l'essor de l'industrie et du commerce, le ministre de l'Intérieur, Chaptal, veut réagir face à la grogne des "artistes" manufacturiers de sa trempe, confrontés à l'arbitraire municipal et la rumeur publique sensible aux nouvelles odeurs. II demande à l'Institut de France, en 1804, « un Rapport de la Classe sur les fabriques qui exhalent une odeur désagréable et sur le danger qu'elles peuvent avoir pour la santé publique. M.M. Chaptal et Guyton ${ }^{10}$ sont chargés de rédiger ce rapport " ", présenté en décembre 1805 (26 frimaire an XIII). L'ambition de Chaptal est d'asseoir les manufactures les plus innovantes - et d'abord les siennes - dans l'espace urbain. La consultation des dignitaires de la science permet de renforcer une idéologie selon laquelle deux classes de métiers enchaînent la ville à son milieu : l'une, ancienne et multiple, dégrade son milieu ; l'autre, récente et unitaire, l'en dégage.

Premier centre industriel de l'Empire, la capitale et ses abords sont particulièrement choyés et surveillés par Napoléon. Lieu des fastes du nouveau pouvoir, Paris est porteuse de l'industrie du luxe, donc des techniques nouvelles à haute valeur ajoutée : dix fabriques de colle forte, six d'acides, six de bleu de Prusse, cinq d'amidon, deux d'ammoniac... À bien regarder, son climat urbain est plutôt délétère : à côté des fabriques fondées sur la transformation organique des matières, aux odeurs ammoniacales,

9 C'est l'avis du comte d'Argout, Rapport à la Chambre des Pairs relatif à la loi du ... (27 avril 1827), Paris, 1827, p. 3. Voir encore l'article de Pierre Claude Reynard, « Public order and privilege. Eighteen-century French roots of environmental regulation ", Technology and culture, 43(1), 2002, pp. 1-28.

10 Chimiste, vice-président de la Société d'encouragement pour l'industrie nationale, administrateur de la Monnaie.

11 Institut de France, Académie des sciences, Procès-verbaux des séances de l'Académie, III (1804-1807), Hendaye, 1913, p. 152, séance du 28 brumaire an XIII. "I Rapport demandé à la classe des Sciences physiques et mathématiques de I'Institut sur... "), Annales de chimie, 54, an XIII, pp. 86-103 et Institut de France, op. cit., séance du 26 frimaire an XIII, pp. 165-168. de nouvelles manufactures qui dégagent des vapeurs acides et sulfureuses cherchent à se développer, non sans mal. L'environnement se dégrade à mesure que s'étend l'Empire, la pollution aérienne est forte, étrange et diversifiée, et de plus en plus constante ; aux fumées humides de la tourbe et du bois s'ajoutent celles noirâtres et "plombées " de la houille; la nappe phréatique est infecte, l'eau dure et toujours plus chargée de sels. Populeuse et trois fois plus dense que Londres et Vienne, ses rivales, la capitale concentre les nouveaux riches et la misère. Mais elle est aussi la ville où la richesse est d'abord foncière, où la rente est littéralement sensible aux fluctuations environnementales : une odeur nauséabonde et persistante pénalise le propriétaire qui veut vendre ; un atelier trop enfumé peut faire déménager les locataires circonvoisins les plus solvables ; à l'opposé, un îlot aéré, verdoyant et calme attire... la salubrité est aussi une valeur foncière.

Le Conseil de salubrité créé le 7 juillet 1801 sous l'impulsion de Cadet de Gassicourt, l'un des chimistes habituellement consultés, est chargé de promouvoir, avec les architectes de la petite voirie et les commissaires de police, la nouvelle urbanité : meilleur éclairage nocturne, contrôle des eaux de surface, réduction des fièvres intermittentes, élimination des eaux croupies, meilleure sécurité. Parmentier en est le président élu, mais Deyeux, pharmacien en chef de Napoléon, ancien compagnon d'Égypte, organisateur de la production de betterave sucrière, professeur de chimie notamment à la faculté de médecine, semble y jouer un rôle prééminent. Le rapport de Chaptal et Guyton-Morveau donne d'un coup une puissance politique à cette institution maintenant sensée évaluer les risques urbains et en aviser le maître de l'espace public.

Dès 1806, Dubois, ordonne que tout nouvel établissement doit faire l'objet d'une demande accompagnée " d'un plan figuré des lieux et des constructions projetées $1{ }^{12}$; cette demande sera soumise à l'enquête "commodo et incommodo ", affichée par les commissaires de police de quartier. Les architectes de la petite voirie ${ }^{13}$ sont chargés de certifier la conformité de l'établissement aux règles de sécurité - cheminée, plancher, toiture, accès. De fait « aucune fabrique ne peut s'établir soit dans Paris, soit aux environs, sans une permission spéciale,

\section{Ordonnance du 12 février 1806.}

13 La petite voirie comprend les rues, places, impasses, à l'exception des routes pénétrantes, des boulevards et des accès aux fortifications qui constituent la grande voirie confiée au service des Ponts et Chaussées ou au Génie dans les places fortes. 


\section{Documents relatifs à l'histoire de la pollution}

laquelle n'est accordée que lorsque des personnes nommées à cet effet se sont transportées sur les lieux et ont constaté si les fours, les fourneaux, les cheminées et généralement tous les bâtiments sont construits de manière à ne donner aucune inquiétude sous le rapport de l'incendie, et si les opérations que le fabricant propose d'exécuter ne sont pas de nature à nuire aux propriétaires voisins $11^{14}$. Le Conseil de Salubrité de la Seine est chargé d'aviser le préfet de Police quant à l'implantation de ces fabriques. II aide aussi les fabricants à adopter des techniques moins polluantes ou énergivores. Le premier rapport enregistré date du 13 mars 1806, signé par Deyeux et Cadet, se réfère à la commune de Taverny où le maire craint que les matières faites " de débris d'animaux rejetés par les manufactures de colles fortes, les triperies, les boyauderies, les boucheries, les tanneries $11^{15}$, en pourrissant sur la terre, n'engendrent des maladies. Les experts lui conseillent de ne les autoriser qu'à 700 mètres (400 toises) des habitations, une des rares distances mentionnées. En 1810, le bilan quinquennal de cette phase expérimentale est très satisfaisant.

En quatre ans, de 1806 à 1810, les arts et les métiers du département de la Seine ont fait l'objet de plus de quatre-vingts rapports : huit pour les fabriques d'acide, sept pour celles du bleu de Prusse et des vernis, six pour celles de colle forte ${ }^{16}$ et d'épuration d'huile, cinq pour les amidonneries, quatre pour les fabriques de soude. Le Ministre consulte à nouveau l'Institut en 1809 à propos de la dangerosité des fabriques. L'académie entreprend le Conseil de Salubrité de la Seine, la seule référence en la

14 « Rapport demandé... », op. cit. note 10, p. 272.

15 Rapport du Conseil de salubrité no 1 du 13 mars 1806. Archives de la Préfecture de Police (Paris).

16 II y a en 1808 dix fabriques de colle forte à Paris. Voir rapport $n^{\circ} 84$ du 10 octobre 1808 et Prunet, Le conseil d'hygiène publique et de salubrité - Sa composition de 1802 à 1935, Archives de la préfecture de Police, 279/20. matière, puis avise le ministre. Le décret impérial est promulgué le 15 octobre 1810, classant les fabriques en trois catégories selon le risque qu'elles font courir au voisinage, ordonnant une enquête préalable à toute implantation nouvelle. II reprend à peu près l'ordonnance du préfet de Police de 1806.

Ailleurs, en province, le décret ne semble pas bien compris. Le ministre y revient un an plus tard, à la fin de 1811, dans une longue lettre aux préfets. " Vous savez que ce décret divise les établissements en trois classes et que ni les uns ni les autres ne peuvent être mis en activité sans une permission de l'autorité administrative "). La durée des affiches de l'enquête qui n'était pas précisée dans le décret et que certains pouvaient fixer à quatre mois comme pour l'ouverture de mines est réduite à un mois. « Que ce projet donne naissance ou non à des oppositions, le certificat des maires de communes dans lesquelles les affiches auront été apposées, devra mentionner cette circonstance $\|^{17}$.

" $\|$ est arrivé quelquefois que des conseils de préfecture ont pris des décisions contraires à des demandes en formation d'établissement, ou en suppression de ceux en activité avant le décret du 15 octobre : ces décisions ont donné lieu à des particuliers de m'écrire, pour me prier de les annuler. Ce n'est point à moi qu'ils auraient dû s'adresser pour obtenir cette annulation. Le décret trace aux parties la marche qu'elles ont à suivre. Elles doivent se pourvoir à la commission du contentieux du conseil d'État, en employant le ministère d'un avocat près ce conseil $\|{ }^{18}$. Montalivet ajoute une nomenclature supplémentaire « qui servira dorénavant de règle aux autorités du département $»$.

17 Lettre du 22 novembre 1811, Circulaires, instructions et autres actes..., II, 1807-1815, Paris, 1832, p. 285.

$18 \mathrm{lbid}$., p. 286. 


\title{
Rapport sur les manufactures qui produisent de mauvaises odeurs Paris 1804 (retranscription commentée)
}

\author{
Jean-Antoine Chaptal \\ Louis-Bernard Guyton-Morveau
}

Rapport sur les manufactures qui produisent de mauvaises odeurs, Paris, 1804

Séance du lundi 26 frimaire an 13.

«M. Chaptal reproduit la conclusion du Rapport suivant sur les manufactures qui produisent de mauvaises odeurs:

" Le Ministre de l'Intérieur vient de consulter la Classe sur une question dont la solution intéresse essentiellement notre industrie manufacturière.

II s'agit de décider si le voisinage de certaines fabriques peut être nuisible la santé.

La solution de ce problème doit paroître d'autant plus importante que, par une suite naturelle de la confiance que méritent les décisions de l'Institut, elle pourra désormais former la base des jugemens du magistrat lorsqu'il s'agit de prononcer entre le sort d'une fabrique et la santé des citoyens.

Cette solution est d'autant plus urgente, elle est devenue d'autant plus nécessaire, que le sort des établissemens les plus utiles, je dirai plus, l'existence de plusieurs arts a dépendu jusqu'ici de simples règlemens de police et que, quelques uns repoussés loin des approvisionnemens, de la main d'œuvre ou de la consommation, par les préjugés, l'ignorance ou la jalousie continuent à lutter avec désavantage contre les obstacles sans nombre qu'on oppose à leur développement. C'est ainsi que nous avons vu successivement les fabriques d'acides, de sel ammoniac, de bleu de Prusse, de bière et les préparations de cuir, reléguées hors l'enceinte des villes, et que chaque jour, ces mêmes établissemens sont encore dénoncés à l'autorité par des voisins inquiets ou par des concurrens jaloux.

Tant que le sort de ces fabriques ne sera pas assuré, tant qu'une législation purement arbitraire aura le droit d'interrompre, de suspendre, de gêner le cours d'une fabrication, en un mot, tant qu'un simple magistrat de police tiendra dans ses mains la fortune ou la ruine du manufacturier, comment concevoir qu'il puisse porter l'imprudence jusqu'à se livrer à des entreprises de cette nature ? Comment a-t-on pu espérer que l'industrie manufacturière s'établît sur des bases aussi fragiles ? Cet état d'incertitude, cette lutte continuelle entre le fabricant et ses voisins, cette indécision éternelle sur le sort d'un établissement, paralyse, rétrécit les efforts du manufacturier, et éteint peu à peu son courage et ses facultés.

\|l est donc de première nécessité pour la prospérité des arts, qu'on pose enfin des limites qui ne laissent plus rien à l'arbitraire du magistrat, qui tracent au manufacturier le cercle dans lequel il peut exercer son industrie librement et

1 Institut de France, Académie des sciences, Procès-verbaux des séances de l'Académie, III (1804-1807), op. cit., p. 191, note 10. 


\section{Rapport sur les manufactures de 1804}

sûrement et qui garantissent au propriétaire voisin qu'il n'y a danger, ni pour sa santé, ni pour les produits de son sol.

Pour arriver à la solution de ce problème important il nous paroît indispensable de jetter un coup d'œil sur chacun des arts qui jusqu'à ce moment ont excité le plus de réclamations.

Pour y parvenir, nous les diviserons en deux Classes : la première comprendra tous ceux dont les opérations laissent échapper dans l'atmosphère, par suite de la putréfaction ou de la fermentation, quelques émanations gazeuses qu'on peut regarder comme incommodes par leur odeur, ou dangereuses par leurs effets.

La seconde classe comprendra tous ceux où l'artiste, opérant par le moyen du feu, développe et dégage en vapeurs ou en gaz, divers principes qui sont plus ou moins désagréables à respirer, et sont réputés plus ou moins nuisibles à la santé.

Dans la première classe, on peut faire entrer le rouissage du lin et du chanvre, la boyauderie, les boucheries, les amidonneries, les tanneries, les brasseries, etc..

Dans la seconde, la distillation des acides, celle des vins, des matières animales, l'art du doreur sur les métaux, les préparations de plomb, de cuivre, de mercure, etc..

Les arts compris dans la $1^{\circ}$ classe, considérés sous le rapport de la santé publique, méritent une attention toute particulière, parce que les émanations qui se dégagent par la fermentation ou la putréfaction sont nuisibles à la santé, dans quelques cas et dans quelques circonstances; par exemple le rovissage qu'on pratique dans des eaux tranquilles ou dans des mares, infecte l'air et tue le poisson. Les maladies qu'il occasionne sont toutes connues et décrites; aussi de sages règlemens ont-ils ordonné, presque partout, que cette opération fût pratiquée hors enceinte des villes, à une certaine distance de toute habitation et dans des eaux dont le poisson n'est pas une ressource pour l'habitant.

Sans doute ces règlemens doiventêtre maintenus, mais comme leur exécution entraîne à son tour quelques inconvéniens, il est à désirer que ce procédé de $M$. $\mathrm{Bral}^{2}$, dont M. M. Monge, Berthollet, Tessier et Molard ${ }^{3}$ ont constaté la supériorité, soit bientôt connu et adopté.

Les autres opérations qu'on exécute sur les végétaux ou sur certains produits de la végétation pour en obtenir des liqueurs fermentées comme dans les brasseries, pour en extraire des couleurs comme dans les fabriques de tournesol, d'orseille et d'indigo, ou pour les dépouiller de quelques uns de leurs principes comme dans les amidonneries, papeteries, etc., ne nous paroissent point de nature à pouvoir exciter une inquiète sollicitude de la part du magistrat; dans tous ces cas, les émanations qui s'élèvent de ces matières en fermentation ne peuvent être dangereuses que dans l'enceinte des vaisseaux et appareils qui les contiennent; elles cessent de l'être du moment qu'elles sont mêlées à l'air extérieur ; il ne faut donc qu'un peu de prudence pour éviter tout danger. D'ailleurs le danger n'est jamais pour les habitans des maisons voisines ; il

2 II s'agit de Bralle, récompensé par le Premier Consul le 17 mai 1804, pour un procédé de rouissage à sec. "Ordre est donné pour mettre en usage sa découverte ".

3 Claude-Louis Berthollet, Gaspard Monge et Pierre-Claude Molard sont commissaires nommés par Chaptal alors ministre de l'Intérieur. Tessier est le rédacteur de l'ordonnance relative au nouveau procédé de rovissage, procédé qui échove. Berthollet, chimiste et Monge, géomètre, sont membres de l'Institut, Molard est directeur du Conservatoire des arts et métiers. 
n'intéresse et ne menace que les ouvriers de la fabrique, de sorte que le règlement qui ordonneroit la translation de ces fabriques au dehors des villes et loin de toute habitation seroit, de la part de l'autorité, un acte à la fois injuste, vexatoire, nuisible aux progrès des arts, et ne remédieroit point au mal qu'entraîne l'opération.

Quelques préparations qu'on extrait des matières animales exigent souvent la putréfaction de ces mêmes matières, comme dans celles qui ont pour objet la fabrication des cordes à boyaux. Mais plus souvent, l'emploi des substances animales expose à voir se corrompre les matières mêmes dont on se sert, par un trop long séjour dans l'atelier, ou par suite d'une température trop chaude; c'est ce qui s'observe surtout dans les teintures en coton rouge, où l'on se sert du sang en abondance. L'infection qu'exhalent ces matières corrompues se répand au loin et forme, pour tout le voisinage, une atmosphère très désagréable à respirer. II est d'une bonne administration de faire renouveler les matières, pour prévenir la corruption, et de faire maintenir assez de propreté dans l'atelier, pour qu'on n'y laisse ni traîner, ni pourrir les résidus des substances animales qu'on y emploie.

Sous ce dernier rapport, les boucheries offrent toujours quelques inconvéniens, mais ils ne sont pas assez graves pour qu'on doive les placer hors des villes et les concentrer sur un seul point, comme des spéculateurs le proposent journellement à l'autorité. Un peu d'attention de la part du magistrat, pour que les bouchers ne répandent pas au dehors le sang et quelques débris des animaux qu'ils égorgent, suffit pour remédier pleinement à tout ce que les boucheries présentent de malsain et de dégoûtant.

La fabrication de la poudrette commence à s'établir dans toutes les grandes villes de la France ${ }^{4}$. L'opération par laquelle on ramène les matières fécales à l'état de poudrette, développe nécessairement et pendant longtems une odeur très désagréable. Les établissemens de cette nature doivent donc être formés dans des lieux bien aérés et éloignés de toute habitation; non que nous regardions les produits gazeux qui s'en exhalent comme nuisibles à la santé, mais on ne peut pas nier qu'ils ne soient incommodes, infects, désagréables, pénibles à respirer et que, sous tous ces rapports, ils ne doivent être écartés de l'habitation des hommes.

Il y a une observation très importante à faire sur la décomposition spontanée des substances animales, c'est que les émanations paroissent en être d'autant moins dangereuses que les matières qui éprouvent la putréfaction sont moins humides; dans ce dernier cas, il se dégage une quantité considérable de carbonate d'ammoniaque qui donne son caractère prédominant aux autres matières qui se volatilisent, et corrige le mauvais effet de celles qui seront délétères. Ainsi la décomposition des matières stercorales en plein air et dans les lieux dont la position et l'inclinaison permettent aux liquides de s'échapper, la décomposition des résidus du cocon du ver à soie, développe une énorme quantité de carbonate d'ammoniaque qui châtre la vertu vénéneuse de quelques autres émanations, tandis que ces mêmes substances, décomposées dans l'eau ou abreuvées de ce liquide, exhalent des miasmes douceâtres et nauséabonds dont la respiration est très dangereuse.

Les arts nombreux dans lesquels le manufacturier produit et répand dans l'air, ensuite de ses opérations et à l'aide du feu, des vapeurs plus ou moins désagréables à respirer, constituent la seconde classe de ceux que nous avons à examiner.

Ceux-ci, plus intéressans que les premiers et bien plus intimement liés à la prospérité de l'industrie nationale sont plus souvent encore l'objet des réclamations portées à la décision des magistrats, et sous ce rapport ils nous ont paru mériter une attention plus particulière.

4 À Paris d'abord, Rouen, Caen, Beauvais, etc. : Sabine Barles, L'invention des déchets urbains. France : 1790-1970, Seyssel, Champ Vallon, 2005, p. 67. 


\section{Rapport sur les manufactures de 1804}

Nous commencerons notre examen par la fabrication des acides.

Les acides dont la préparation peut exciter quelques plaintes de la part des voisins de la fabrique, sont le sulfurique, le nitrique, le muriatique et l'acéteux.

Le sulfurique s'obtient par la combustion d'un mélange de soufre et de salpêtre. II est bien difficile que, dans cette opération, il ne se répande une odeur plus ou moins marquée d'acide sulfureux, autour de l'appareil dans lequel s'opère la combustion, mais dans les fabriques conduites avec intelligence, cette odeur est à peine sensible dans l'atelier, elle ne présente aucun danger pour les ouvriers qui la respirent journellement et aucune plainte de la part des voisins ne sauroit être fondée.

Lorsque l'art de fabriquer l'acide sulfurique a été introduit en France, l'opinion publique s'est fortement prononcée contre les premiers établissemens ${ }^{5}$; l'odeur de l'allumette qu'on brûle dans nos foyers ne contribuoit pas peu à exagérer l'effet que devoit produire la combustion rapide de quelques quintaux de soufre ; aujourd'hui l'opinion est si bien revenue sur leur compte que nous voyons plusieurs de ces fabriques prospérer paisiblement et sans trouble au milieu de nos villes ${ }^{6}$.

La distillation des eaux fortes et de l'esprit de sel (acide nitrique et muriatique) ne présente pas plus de danger que la fabrication de l'acide sulfurique; toute l'opération se fait dans des appareils de grès ou de verre, et le premier intérêt du fabricant est, sans contredit, de diminuer la déperdition ou la volatilisation autant qu'il est en son pouvoir ; cependant quelque attention qu'on donne au procédé, l'air qu'on respire dans l'atelier est toujours imprégné de l'odeur particulière à chacun de ces acides. Néanmoins la respiration y est libre et sûre, les hommes qui y travaillent journellement n'y sont pas du tout incommodés et les voisins auroient grand tort de se plaindre.

Depuis que les fabriques de blanc de plomb, de vert de gris et de sel de Saturne se sont multipliées en France, le vinaigre y est d'un usage plus général.

Lorsqu'on distille cet acide pour le rendre propre à quelques uns de ces usages, il se répand au loin une odeur très forte de vinaigre, qui ne présente aucun danger. Mais lorsqu'on évapore une dissolution de plomb dans cet acide, les vapeurs prennent alors un caractère douceâtre, et produisent sur les hommes qui les respirent habituellement tous les effets particuliers aux émanations du plomb lui-même; heureusement que ces effets n'affectent que les ouvriers qui travaillent dans l'atelier et qu'ils sont insensibles pour toutes les personnes qui vivent dans le voisinage.

Les préparations de mercure et de plomb, celles de cuivre, d'antimoine et d'arsenic, les opérations du doreur sur métaux présentent presque toutes, quelque danger pour les personnes qui habitent dans les ateliers et concourent aux opérations, mais les effets se bornent dans l'enceinte des ateliers; tout y est pour ainsi dire aux risques et périls des entrepreneurs et fabricans. II est digne des chimistes de s'occuper des moyens de prévenir ces fâcheux résultats ; déjà même on a obvié à plusieurs inconvéniens à l'aide de cheminées qui aspirent les vapeurs et les portent dans les airs, hors de toute atteinte pour la respiration, et aujourd'hui toute l'attention de l'administration doit se borner à diriger les sciences vers les moyens de perfectionnement dont ces procédés sont susceptibles sous le rapport de la santé.

5 Notamment à Montpellier où Chaptal a installé sa première fabrique en 1784 . 6 L'enquête de 1806 révèle une dizaine de fabriques à Paris, Rapport du Conseil de salubrité de la Seine $n^{\circ} 11$ du 5 août 1806, Archives de la Préfecture de Police (Paris). 
La fabrication du bleu de Prusse, l'extraction du carbonate d'ammoniaque par la distillation des matières animales dans les nouvelles fabriques de sel ammoniac, produisent une grande quantité de vapeurs ou exhalaisons fétides. À la vérité ces exhalaisons ne sont pas dangereuses pour la santé ; cependant, comme pour être bon voisin, il ne suffit pas de n'être pas dangereux et qu'il faut encore n'être pas incommode, les entrepreneurs de ces sortes d'établissemens, lorsqu'ils ont à se déterminer sur le choix d'un emplacement, doivent préférer celui qui est éloigné de toute habitation. Mais lorsque l'établissement est déjà formé, nous nous garderons bien de conseiller au magistrat d'en ordonner la translation; il suffit dans ce cas d'exiger de l'entrepreneur qu'il construise des cheminées très élevées pour noyer dans les airs les vapeurs désagréables qui sont produites dans ces opérations. Ce moyen est surtout praticable pour la fabrication du bleu de Prusse, et c'est en pratiquant que I'un de nous a fait conserver au milieu de Paris une des fabriques les plus importantes dans ce genre, contre laquelle les voisins et l'autorité s'étoient déjà ligués?.

Dans le Rapport que nous soumettons à la Classe nous n'avons cru devoir nous occuper que des principales fabriques contre lesquelles de violentes réclamations se sont élevées, en divers tems et en divers lieux. II est aisé de voir, d'après ce qui précède, qu'il en est peu dont le voisinage soit nuisible à la santé.

D'après cela nous ne saurions trop inviter les magistrats chargés de la santé et sûreté publiques, d'écarter les plaintes mal fondées qui, trop souvent, se dirigent contre les établissemens, menacent chaque jour la fortune de l'honnête manufacturier, retardent les progrès de l'industrie et compromettent le sort de l'art lui-même.

Le magistrat doit être en garde contre les démarches d'un voisin inquiet ou jaloux : il doit distinguer avec soin ce qui n'est qu'incommode ou désagréable, d'avec ce qui est nuisible ou dangereux ; il doit se rappeler qu'on a proscrit pendant longtems l'usage de la houille, sous le prétexte frivole qu'elle étoit malsaine; il doit, en un mot, se pénétrer de cette vérité, c'est qu'en accueillant les plaintes de cette nature, non seulement on parviendroit à empêcher l'établissement en France de plusieurs arts utiles, mais on arriveroit insensiblement à éloigner des villes les maréchaux, les charpentiers. les menuisiers, les chaudronniers, les tonneliers, les fondeurs, les tisserans et généralement tous ceux dont la profession est plus ou moins incommode pour le voisin. À coup sûr, les arts que nous venons de nommer forment un voisinage plus désagréable que celui des fabriques dont nous avons parlé ; le seul avantage qu'ils ont sur ces dernières, c'est leur ancienneté d'exercice ; leur droit de domicile s'est établi avec le temps et par le besoin : ne doutons pas que, lorsque nos fabriques seront plus vieilles et mieux connues, elles ne jouissent paisiblement du même avantage dans la société. En attendant, nous pensons que la Classe doit profiter de cette circonstance, pour les mettre d'une manière spéciale sous la protection du Gouvernement et déclarer que les fabriques d'acides, de sel ammoniac, de bleu de Prusse, de sel de Saturne, de blanc de plomb, les boucheries, les amidonneries, les brasseries, les tanneries, ne forment point un voisinage nuisible à la santé, lorsqu'elles sont bien conduites.

Nous ne pouvons pas en dire autant du rouissage du chanvre, des boyauderies, des voieries et généralement de tous les établissemens où l'on soumet une grande quantité de matières animales ou végétales à une putréfaction humide; dans tous ces cas, outre l'odeur très désagréable qui s'exhale, il se dégage encore des miasmes qui sont plus ou moins malfaisans.

7 II s'agit d'une fabrique implantée rue Saint-Nicolas, dans le faubourg Saint-Martin, Rapport du Conseil de salubrité, op. cit., nº 18 du 15 février 1807. 


\section{Rapport sur les manufactures de 1804}

Nous devons ajouter que, quoique les fabriques dont nous avons déjà parlé, et que nous avons considérées comme n'étant pas nuisibles à la santé par leur voisinage ne doivent pas être déplacées, néanmoins l'administration doit être invitée à exercer sur elles la surveillance la plus active et à consulter les personnes instruites, pour prescrire aux entrepreneurs les mesures les plus propres à empêcher que les odeurs et la fumée ne se répandent dans le voisinage. On peut atteindre ce but en améliorant les procédés de fabrication, en élevant les murs d'enceinte pour que la vapeur ne soit pas déversée sur les habitations voisines, en perfectionnant la conduite du feu, qui peut être telle que la fumée elle-même soit brûlée dans les foyers ou déposée dans les longs tuyaux des cheminées, en entretenant la plus grande propreté dans les ateliers, de manière qu'aucune matière ne s'y corrompe et que tous les résidus susceptibles de fermentation aillent se perdre dans des puits profonds et ne puissent en aucune manière incommoder les voisins.

Nous observerons encore que, lorsqu'il s'agit de former de nouveaux établissemens de bleu de Prusse, de sel ammoniac, de tanneries, d'amidonneries et généralement de toute fabrication qui produit nécessairement des vapeurs très incommodes pour les voisins ou des dangers toujours renaissans par la crainte du feu ou des explosions, il seroit à la fois sage, juste et prudent de prononcer en principe que ces établissemens ne pourroient être formés dans l'enceinte des villes et près des habitations qu'avec une autorisation spéciale et que, dans le cas où les entrepreneurs ne rempliroient pas cette condition indispensable, la translation de leurs établissemens pourroit être ordonnée sans indemnité.

II résulte donc de notre Rapport $1^{\circ}$ que les établissemens de boyauderies, de rouissage, de voierie et généralement tous ceux dans lesquels on amoncelle et fait pourrir et putréfier en grande masse des matières animales ou végétales, forment un voisinage nuisible à la santé et qu'on doit les porter hors l'enceinte des villes et de toute habitation ; $2^{\circ}$ que les fabriques dans lesquelles on développe les odeurs désagréables par le moyen du feu, comme dans la fabrication des acides, du bleu de Prusse, du sel ammoniac ne forment un voisinage dangereux que par défaut de précaution et que les soins de l'administration doivent se borner à une surveillance active et éclairée, pour faire perfectionner les procédés dans la fabrication et la conduite du feu, et pour y maintenir une propreté convenable ; $3^{\circ}$ qu'il seroit digne d'une bonne et sage administration de faire des règlemens qui prohibassent pour l'avenir, dans l'enceinte des villes et près des habitations, l'établissement de toute fabrique dont le voisinage est essentiellement incommode ou dangereux, sans une autorisation préalable. On peut comprendre dans cette classe les poudreries, les tanneries, les amidonneries, les fonderies de métal et de suif, les boucheries, les amas de chiffons, les fabriques de bleu de Prusse, de vernis, de colle forte, de sel ammoniac, de poteries etc..

Telles sont les conclusions que nous avons l'honneur de soumettre à la Classe.

Signé à la minute : Guyton, Chaptal. La Classe approuve le Rapport et en adopte les conclusions.

184 Documents pour l'histoire des techniques $\mathrm{n}^{\circ} 17$ - juin 2009 


\title{
Ordonnance du 12 février 1806 concernant les ateliers, manufactures ou laboratoires
}

\author{
(retranscription)
}

\section{Préfecture de Police}

Préfecture de Police. Ordonnance du 12 février 1806 concernant les ateliers, manufactures ou laboratoires'

Paris, le 12 février 1806.

Le Conseiller d'État, chargé du $4^{e}$ arrondissement de la Police générale de l'Empire, Préfet de Police, et l'un des commandans de la Légion d'honneur,

Considérant qu'il s'établit journellement dans la ville de Paris, des Ateliers, Manufactures ou Laboratoires qui, soit par la nature des matières qu'on y travaille, soit par l'usage du feu qu' on y fait, soit enfin par le défaut de précautions suffisantes, peuvent compromettre la salubrité et occasionner des incendies ;

Considérant que ces sortes d'établissements excitent des plaintes qui obligent l'autorité d'en prononcer la suppression ou d'en suspendre l'activité, et qu'il en résulte souvent que les frais considérables auxquels ils ont donné lieu, deviennent en pure perte pour les propriétaires;

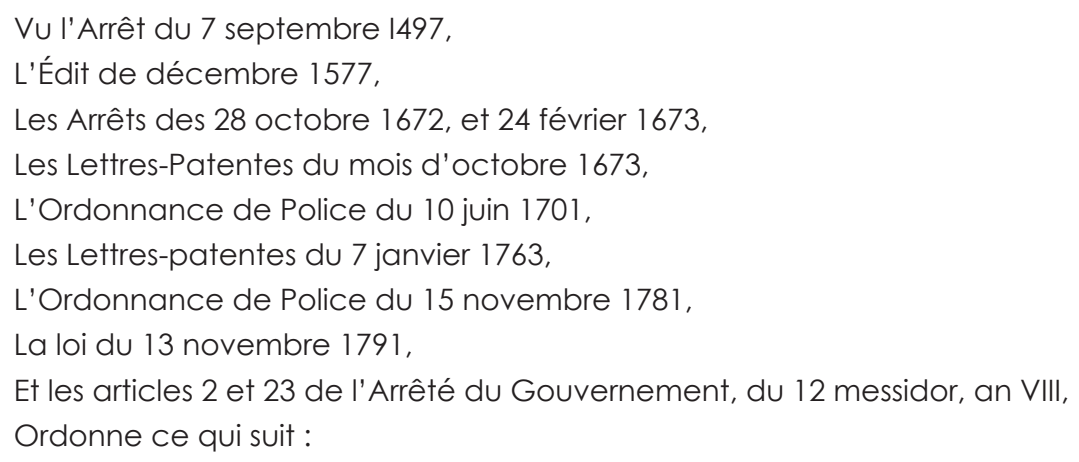

1 Préfecture de Police, Recueil des décrets et ordonnances du Préfet de Police, Paris, 1832, I, p. 124. 
ARTICLE PREMIER.

IL est défendu d'établir dans la ville de Paris, aucun Atelier, Manufacture ou Laboratoire qui pourraient compromettre la salubrité ou occasionner un incendie, sans avoir préalablement fait à la Préfecture de Police, la déclaration de la nature des matières qu'on se proposera d'y préparer, et des travaux qui devront y être exécutés.

Il sera déposé en même-temps un plan figuré des lieux et des constructions projettées.

Art. II. AUSSI-TÔT après cette déclaration il sera procédé par des gens de l'art, assistés d'un Commissaire de Police, à la visite des lieux, à l'effet de s'assurer si l'établissement projetté ne peut point nuire à la salubrité ni faire craindre un incendie. II en sera dressé procès-verbal d'enquête de commodo et incommodo, pour être statué ce qu'il appartiendra.

ART. III. LA présente Ordonnance sera imprimée, publiée et affichée.

Les Commissaires de Police. I'Inspecteur-général du $4^{\text {e }}$ arrondissement de la Police générale de l'Empire, les Officiers de Paix, l'Architecte-Commissaire de la petite Voirie, l'Inspecteur-général de la Salubrité, les Commissaires des Halles et Marchés, et les autres Préposés de la Préfecture de Police sont chargés, chacun en ce qui le concerne de tenir la main à son exécution.

Le Conseiller d'État, Préfet, Signé, DUBOIS. 


\title{
Institut de France \\ Séance du lundi 30 octobre 1809
}

\author{
(retranscription)
}

\section{Institut de France}

Institut de France. Séance du lundi 30 octobre 18091

" Au nom d'une Commission, M. Deyeux lit le Rapport suivant sur les manufactures de produits chimiques qui peuvent être dangereux :

« En comparant les fabriques qui existoient il y a 20 ans avec celles qui aujourd'hui sont en activité, on est frappé de l'amélioration que les procédés qu'on suit dans ces dernières ont éprouvée, et en même tems on est forcé de convenir qu'elles doivent cet avantage aux lumières qu'elles ont empruntées de la chimie et de l'heureuse application qu'elles ont su en faire.

Par une conséquence naturelle de cet état de choses, le nombre des fabriques a dû nécessairement augmenter, et l'industrie nationale, en se perfectionnant, a dû nécessairement aussi donner lieu à de nombreuses spéculations dont les résultats sont devenus d'autant plus avantageux qu'ils ont tourné au profit de la Société.

Mais si d'un côté on doit savoir gré aux fabricans du zèle qu'ils mettent à poursuivre leurs travaux et à les multiplier, ainsi que des sacrifices que souvent ils font avant même d'avoir acquis la certitude d'obtenir des succès, on a aussi quelques reproches à leur faire sur l'insouciance avec laquelle plusieurs d'entr'eux choisissent les localités où ils établissent leurs fabriques.

Uniquement occupés de l'emploi des moyens qui doivent leur procurer les résultats qu'ils désirent obtenir, ils ne cherchent pas toujours à s'assurer si les matières premières dont ils se servent ou les produits qu'ils en séparent donnent, pendant leur traitement, naissance à des vapeurs d'une odeur désagréable qui, en se répandant plus ou moins promptement et à des distances plus ou moins éloignées, finissent par incommoder ceux qui les respirent.

C'est sans doute à ce peu de précautions ou à cet oubli qu'on doit attribuer les plaintes formées contre certaines fabriques, et les demandes réitérées tendant à obtenir leur suppression ou au moins leur éloignement des lieux environnés d'habitations.

S'il est impossible de ne pas reconnoître souvent la justesse de ses plaintes, on est aussi forcé de convenir que quelquefois elles n'ont pour véritable prétexte que des inquiétudes mal fondées, des préventions, des jalousies et des rivalités. Il devenoit donc nécessaire de chercher des moyens qui, en dissipant à cet égard toute espèce d'incertitude, fixassent d'une manière sûre et constante les bases sur lesquelles devoient être établies les décisions des magistrats devant qui les plaintes étoient portées.

Déjà, en l'an 13, le Ministre de l'Intérieur, convaincu des difficultés que présentoit un travail fait d'après ces vues, avoit écrit à la Classe des Sciences physiques et mathématiques pour l'inviter à s'occuper de cet objet important.

1 Institut de France, Académie des sciences, Procès verbaux des séances de l'Académie, IV (1808-1811), Hendaye, 1915, pp. 268-273. 
Les Commissaires qui, à cette époque, furent nommés, rédigèrent un Rapport dans lequel ils proposoient plusieurs mesures qu'ils croyoient qu'on devoit prendre, et indiquoient surtout les manufactures ou fabriques qui leur paroissoient devoir être conservées, et celles qu'il convenoit d'éloigner du voisinage des lieux habités.

Ce Rapport fait avec beaucoup de soin et rempli d'observations très intéressantes et judicieuses a été unanimement adopté par la Classe, et a souvent guidé le Magistrat de Police, soit lorsqu'il croyoit devoir faire droit aux réclamations qui lui étoient présentées, soit lorsqu'il jugeoit convenable de les écarter.

Malheureusement l'expérience ne tarda pas à prouver que ce Rapport, qui d'abord avoit paru suffisant pour remplir les vues du Ministre, n'offrant que des données générales, étoit susceptible de différentes interprétations qui, suivant qu'elles étoient plus ou moins favorables aux réclamans et aux fabricans, donnoient lieu à de nouvelles plaintes que les partis qui se croyoient lésés poursuivoient avec chaleur.

Voulant faire disparoître ces inconvéniens, le Ministre s'est de nouveau adressé à la première Classe de l'Institut, et après avoir exposé dans une lettre très détaillée les motifs qui l'engagent à réclamer encore son avis, il l'invite à prendre sa demande en grande considération.

La Classe, à son tour, convaincue de l'importance de l'affaire qui lui étoit soumise, a pensé quelle devoit charger du soin de l'examiner ceux de ses Membres qui, par la nature de leurs travaux particuliers, étoient portés de connoître, non seulement les divers produits que les fabriques fournissent au commerce, mais même encore les opérations employées pour obtenir ces produits. En conséquence, elle a arrêté que la Section de Chimie seroit invitée à présenter incessamment un Rapport sur la demande du Ministre.

Le premier soin de la Commission a été de bien se pénétrer des diverses observations insérées dans la lettre du Ministre ; elles méritoient en effet de fixer d'autant plus l'attention qu'elles présentoient un aperçu des motifs qu'on pouvoit faire valoir pour éloigner certaines fabriques et en conserver d'autres.

Voici, à cet égard comment le Ministre s'est exprimé :

" S'il est juste, est-il dit dans sa lettre, que chacun puisse exploiter librement son industrie, le Gouvernement ne sauroit d'un autre côté voir avec indifférence que, pour l'avantage d'un individu, tout un quartier respire un air infecté, ou qu'un particulier éprouve des dommages dans sa propriété. En admettant que la plupart des manufacturiers dont on se plaint n'occasionnent pas d'exhalaisons contraires à la salubrité publique, on ne niera pas non plus que ces exhalaisons peuvent être quelquefois désagréables et que, par cela même, elles ne portent un préjudice réel aux propriétaires des maisons voisines, ou en les forçant, s'ils les lovent, à baisser le prix de leurs baux. Comme la sollicitude du Gouvernement embrasse toutes les Classes de la société, il est de sa justice que les intérêts de ces propriétaires ne soient pas perdus de vue plus que ceux des manufacturiers. II paroîtra peut-être, d'après cela, convenable d'arrêter en principe que les établissemens qui répandent une odeur forte et gênent la respiration ne seront dorénavant formés que dans les localités isolées. ॥ 
II étoit difficile de se refuser à l'évidence de principes aussi incontestables que ceux établis dans le paragraphe de la lettre qu'on vient de citer. Aussi la Commission s'est-elle empressée de les adopter et de les considérer comme devant servir de base aux différentes propositions qu'elle auroit à faire.

Toutes les fabriques variant entr'elles par la nature des travaux qui les occupent, il étoit nécessaire de se procurer une connoissance exacte de celles qui, étant en activité surtout dans le ressort de Paris, devoient principalement fixer l'attention.

Pour cela la Commission s'est adressée à M. le Préfet de police qui, sur le champ, a donné des ordres dans ses bureaux pour qu'il fût rédigé un tableau de tous les ateliers, fabriques et établissemens qui sont sous sa surveillance.

C'est d'après ce tableau que la Commission a opéré et qu'elle a arrêté qu'il seroit divisé en trois Classes, dont la première comprendroit les établissemens ou fabriques qui décidément devoient être éloignées des endroits habités ; la seconde, ceux de ces établissemens qui, pouvant rester auprès des habitations, avoient cependant besoin d'être surveillés, et enfin, la troisième, ceux qui pouvoient être placés partout et dont le voisinage n' offroit aucun inconvénient, soit sous le rapport de la sûreté, soit sous celui de la salubrité.

En lisant ce tableau qui se trouve annexé au présent Rapport, on sera bientôt convaincu :

$1^{\circ}$ Que les établissemens compris dans la première classe ne doivent pas rester auprès des habitations puisque les matières qu' on y travaille et les produits qu'on en retire, ou répandent une odeur désagréable qu'il est difficile de supporter et qui nuit à la Salubrité, ou sont susceptibles de compromettre la sûreté publique par des accidens auxquels ils pouvoient donner lieu. Ainsi, par exemple, les boyauderies dans lesquelles on rassemble les intestins de divers animaux pour leur faire subir des préparations qui les amènent à cet état particulier où ils doivent être pour permettre qu'ensuite on les emploie à différens usages; les fabriques de colle forte dans lesquelles on ne se sert que de débris d'animaux qu'on fait macérer dans l'eau jusqu'à ce qu'ils aient éprouvé une fermentation putride très avancée et qu'on croit nécessaire pour obtenir la substance qui forme la colle; les amidonneries dans lesquelles aussi les grains, les sons, les recoupes, les riots doivent indispensablement être soumis à la fermentation putride ; les ateliers d'êquarrissage et de poudrette, tous ces établissemens et beaucoup d'autres de cette espèce, considérés sous le rapport de la salubrité, ne peuvent et ne doivent pas, à cause de la mauvaise odeur qu'ils répandent, être placés près des habitations. En vain essaie-t-on de prouver par de simples raisonnemens l'innocuité des gaz qui proviennent de ces fabriques; jamais on ne parviendra à persuader qu'on peut les respirer impunément et que l'air qui les contient n'est pas aussi insalubre qu'on le croit.

Par d'autres raisons, non moins essentielles, on a dû placer dans la première classe des fabriques qu'il convient d'éloigner, celles qui peuvent compromettre la sûreté publique. Tels sont entr'autres les ateliers des artificiers et les poudrières qui, malgré toutes les précautions que prennent ceux qui les dirigent, sont susceptibles d'une foule d'inconvéniens dont malheureusement on n'a que trop d'exemples. Au reste, en demandant l'éloignement des fabriques dont il vient d'être question, on ne fait pour ainsi dire que réclamer l'exécution d'anciennes ordonnances de police qui n'ont jamais été abrogées et d'après lesquelles 
il est constant qu'il y avoit certaines fabriques qu'on ne souffroit jamais dans l'intérieur de la ville. Si alors on se contentoit de les reléguer dans les faubourgs, c'est que les faubourgs, qui étoient peu peuplés, offroient de vastes terrains inhabités sur lesquels les fabricans pouvoient établir des ateliers sans craindre que leur voisinage pût devenir incommode aux plus proches voisins.

Mais aujourd'hui que les fabriques se sont multipliées et que dans les faubourgs les maisons particulières sont presque en aussi grand nombre et presque aussi resserrées que dans l'intérieur de la ville, on ne voit plus sans inquiétudes de nouvelles fabriques s'y élever, et si on supporte celles qui existent depuis longtems, c'est que les propriétaires des maisons qui ont été bâties depuis n'ont pas droit de se plaindre, puisqu'ils ont dû s'attendre aux inconvéniens auxquels les exposoit le voisinage de ces établissemens.

Quoique, d'après ce qui vient d'être dit, la nécessité d'écarter toutes les fabriques comprises dans la première classe du tableau paroisse bien démontrée, la Commission doit néanmoins faire observer qu'elle n'est pas éloignée de croire à la possibilité d'en pouvoir diminuer le nombre par la suite, surtout si les fabricans, abandonnant quelques uns des procédés qu'ils emploient aujourd'hui, parviennent à en découvrir d'autres qui, sans avoir les mêmes inconvéniens que ceux dont ils se servent, n'en soient pas moins propres à leur procurer les résultats qu'ils cherchent à obtenir.

Déjà même on sait que, dans quelques fabriques de soude et de bleu de Prusse, dont le voisinage est si redoutable lorsqu'on emploie les procédés ordinaires, on commence à faire usage d'opérations nouvelles au moyen desquelles les gaz acide muriatique et hydrogène sulfuré sont si bien coercés, absorbés ou dilatés qu'à peine même sont-ils sensibles dans l'intérieur des fabriques ; mais il reste à savoir si ces opérations faites en grand auront du succès et si leur emploi n'est pas lui-même sujet à quelques inconvénens.

$2^{\circ}$ Les ateliers, établissemens et fabriques compris dans la seconde classe du tableau n'ont pas été jugés par la Commission être dans le cas qu'on exigeât qu'ils fussent aussi éloignés des lieux habités que ceux compris dans la première classe ; mais cependant elle a pensé qu'il étoit indispensable de les surveiller.

Pour bien sentir les motifs de cette opinion, il suffit de savoir que la plupart des opérations qui se pratiquent dans ces établissemens ne peuvent produire de vapeurs nuisibles qu'autant qu'on ne prend pas tous les soins qui conviennent pour opérer leur condensation. Or comme les procédés et les appareils au moyen desquels on parvient aisément à s'en rendre maître sont aujourd'hui parfaitement connus et généralement adoptés, on n'a besoin que de recommander qu'ils soient employés, et il est indubitable qu'ils le seront, lorsque les propriétaires des fabriques dont il s'agit sauront qu'on les surveille et que la moindre négligence de leur part pourroit les exposer à recevoir l'ordre de cesser leurs travaux.

Il faut cependant convenir que dans plusieurs des fabriques comprises dans cette seconde classe, telle précaution qu'on prenne pour bien lutter ces appareils, il y a toujours des gaz qui se séparent et qui sans doute incommoderoient les voisins si leur quantité n'étoit pas si peu considérable, que rarement ils dépassent l'intérieur des ateliers. Aussi les ouvriers qui y travaillent seroient-ils les seuls fondés à s'en plaindre, si l'habitude de les respirer ne les rendoit pas pour ainsi dire insensibles à leur action.

C'est ainsi par exemple que lorsqu'on entre dans les fabriques d'acide sulfurique, nitrique et muriatique simple et oxigéné. on est frappé tout à coup 
de l'odeur de ces acides, tandis que les ouvriers s'en aperçoivent à peine et qu'ils n'en sont incommodés que quand, faute de prévoyance, ils en respirent beaucoup à la fois.

Au surplus, peut-être seroit-il prudent d'exiger que surtout les grandes fabriques d'acides fussent placées à l'extrémité des villes dans les quartiers peu peuplés, et qu'elles fussent disposées de manière que, dans le cas où quelques gaz viendroient à s'en échapper, ils pussent être entraînés sur le champ par des courans d'air. Cette précaution suffiroit pour mettre les voisins à l'abri de toute espèce d'inquiétude.

$3^{\circ}$ Quant aux établissemens indiqués dans la $3^{\circ}$ classe, la Commission est d'avis qu'il y a d'autant moins d'inconvénient à permettre qu'ils soient placés près des habitations, que sous aucun rapport ils ne peuvent être nuisibles, et que les précautions qu'on a droit d'exiger des propriétaires de ces établissemens sont les mêmes que celles que tous les individus qui vivent en société prennent ordinairement lorsqu'ils ne veulent pas se nuire réciproquement.

Reste maintenant à s'occuper d'une demande que le Ministre a faite et qui est relative à la distance des habitations que doivent observer les fabriques dont l'éloignement est jugé nécessaire et indispensable.

La Commission ne doit pas dissimuler qu'en méditant sur cette demande, elle s'est trouvée fort embarrassée pour y répondre.

En effet, on conçoit facilement que toutes les localités n'étant pas les mêmes, si on établissoit la distance où doivent être placées les manufactures des lieux habités, il en résulteroit que souvent un local assez voisin d'habitations pourroit cependant par la nature même de sa position, convenir à l'établissement d'une manufacture, Sans que les habitans des maisons les plus voisines fussent dans le cas de s'apercevoir des vapeurs qui s'exhaleroient de cet établissement. Ainsi par exemple, on suppose un local placé dans un fond et environné du côté des endroits habités par de hautes montagnes. Assurément, un local semblable, quoique voisin d'habitations, n'offriroit aucun inconvénient pour y placer une fabrique, puisque les vapeurs avant de parvenir au sommet des montagnes auroient été forcées de traverser une grande masse d'air atmosphérique ou elles auroient perdu en se dissolvant toute leur propriété insalubre.

Cette supposition qu' on cite pour exemple paroîtra d'autant moins déplacée qu'il est possible de la justifier par un fait dont un des Membres de la Commission vient tout récemment d'être témoin. Ce fait mérite d'être cité. Un fabricant de soude artificielle, après avoir été obligé de quitter un emplacement dans lequel il avoit fait ses premiers essais parce que ses voisins se plaignoient de la vapeur acide à laquelle ils étoient exposés, crut avoir trouvé un endroit qui ne seroit pas sujet au même inconvénient que le premier, en se plaçant dans le fond d'une profonde carrière abandonnée qui, d'un côté, est bordée de montagnes de la hauteur de 88 mètres à partir du sol de la carrière, et dont le côté opposé donne sur la campagne ${ }^{2}$. Quelques habitans des maisons construites sur le plateau

2 Suite aux plaintes réitérées du maire du Petit Gentilly et des cabaretiers voisins, la fabrique d'acides de Marc et Costel placée à Maison-Blanche déménage, après autorisation préfectorale, en septembre 1809, à Belleville entre la voirie de Montfaucon et « la sape de la montagne à plâtre dont l'exploitation est abandonnée depuis plusieurs années ». Le site est éloigné des habitations et « la mauvaise qualité du terrain n'est pas favorable à la culture et à la végétation... En plus les émanations d'acide muriatique seront en quelque sorte annihilées en se combinant avec les gaz ammoniacaux et l'acide sulfureux qui, sans cesse, s'élèvent du réservoir immense où sont déposées les matières fécales, gaz qui, comme 
de ces montagnes conçurent des inquiétudes lorsqu'ils apprirent qu'on alloit s'occuper de l'établissement projeté. Ils mirent aussitôt tout en œuvre pour s'y opposer et ils vinrent à bout, à force de tracasseries, à déterminer le fabricant à abandonner le local qu'il avoit choisi, quoique, sous beaucoup de rapports, il eût dû lui convenir.

Une autre raison encore qui prouve la difficulté d'établir dans un règlement d'une manière exacte la distance qu'on doit assigner aux fabriques qui sont dans le cas d'être éloignées, c'est que, les gaz qu'elles répandent n'étant ni de même nature, ni également expansibles, ni délétères au même degré, il ne seroit pas raisonnable d'exiger qu'elles fussent toutes également forcées à isoler des villes ou des lieux habités. Or, comme pour fixer les limites de chaque fabrique il faudroit avoir des renseignemens positifs, tant sur les localités que sur l'extension plus ou moins grande que chaque fabrique voudroit donner à ses travaux et qu'on ne peut pas se les procurer facilement, il en résulte qu'une fixation exacte des distances que doivent observer les fabriques est presque impossible. Cependant pour se tirer d'embarras, la Commission a pensé qu'on pourroit adopter provisoirement les moyens suivans qui consistent à établir en principe général que toutes les fabriques comprises dans la première classe du tableau ne pourront être placées qu'à des distances assez éloignées des villes pour ne pas incommoder les habitans des maisons les plus voisines, et que, quant au surplus, on s'en rapportera aux autorités chargées de la surveillance et de la police des fabriques, attendu que par la nature de leurs fonctions, elles sont plus à portée que personne de se procurer des informations sur les avantages ou sur les inconvéniens que pourront présenter les localités où les fabricans voudront s'établir.

À ces moyens on pourroit encore ajouter la précaution d'exiger de tout fabricant qui voudra s'établir une déclaration de l'endroit où il a l'intention de se placer, ainsi que du genre d'opérations qu'il se propose de suivre, et ne lui accorder la permission de commencer ses travaux qu'après l'avoir prévenu que dans le cas où il surviendroit des plaintes contre lui, plaintes qui seroient constatées par des personnes en état de juger si elles sont légitimes, il lui seroit enjoint de fermer sa fabrique et de la porter ailleurs. On seroit bien sûr alors que le fabricant qui ne voudroit pas courir le risque de perdre les dépenses qu'il auroit faites ne manqueroit pas de choisir un emplacement où il seroit à l'abri de tout reproche. La Commission est d'autant plus fondée à croire aux succès des moyens qui viennent d'être proposés, que déjà l'expérience a prononcé en leur faveur. Pour en avoir la preuve, il suffit de savoir que depuis trois ans environ, aucune fabrique ne peut s'établir soit dans Paris, soit aux environs, sans une permission spéciale laquelle n'est accordée que lorsque des personnes nommées à cet effet se sont transportées sur les lieux et ont constaté si les fours, les fourneaux, les cheminées et généralement tous les bâtimens sont construits de manière à ne donner aucune inquiétude sous le rapport de l'incendie, et si les opérations que le fabricant

on sait, s'étend à de très grandes distances et incommodent les maisons les plus voisines de Montfaucon... [On aurait ainsi] une sorte de fumigation guytonienne qui contribuerait à assainir une étendue de terrain qui n'est point habitable dans certain temps de l'année ", Rapports du Conseil de salubrité au Préfet de Police signés de Deyeux n 150 du 3 septembre 1809 et nº162 du 30 septembre, Archives de la Préfecture de Police. André Guillerme, AnneCécile Lefort, Gérard Jigaudon, Dangereux, insalubres et incommodes : paysages industriels en banlieve parisienne (XIXe-XXe siècles), Seyssel, Champ Vallon, 2005, p. 97. 
propose d'exécuter ne sont pas de nature à nuire aux propriétaires voisins.

C'est, on le répète, avec de semblables mesures qu'on est parvenu à éloigner plusieurs fabriques qui, si elles eussent été placées où on voulait les établir n'auraient pas manqué de donner lieu à des plaintes bien fondées et auxquelles par conséquent il aurait été impossible de ne pas faire droit sans commettre une injustice.

De toutes les fabriques actuellement existantes, celles où depuis quelque tems on s'occupe de l'extraction de la soude en décomposant le sel marin ${ }^{3}$, ont excité de vives réclamations qui, malheureusement ne sont que trop fondées. Pour s'en convaincre, il suffit de savoir qu'il est de notoriété publique que presque toutes les propriétés voisines de ces fabriques ont été si endommagées, qu'il a fallu souvent les abandonner. On cite même, entr'autres choses, des récoltes entières dans l'étendue à peu près d'un quart de lieve de rayon qui ont été entièrement détruites.

Assurément des fabriques de cette espèce doivent être plus éloignées que d'autres, et les localités qui leur conviennent sont celles qui, à une très grande distance, sont environnées de terrains inhabités et incultes. Cependant cette condition ne devrait être de rigueur qu'autant que les fabricans de soude artificielle persisteront à se servir du procédé qu'ils ont employé jusqu'ici pour se débarrasser de l'acide muriatique qu'ils dégagent du sel marin ; car si, comme on l'a déjà dit, ils en trouvaient un autre au moyen duquel ils parvinssent à s'opposer à l'évaporation de acide, il n'y auroit plus alors le moindre doute que les fabriques de soude pourront être assimilées à beaucoup d'autres qui n'exigent pas un éloignement très considérable des lieux habités.

D'après toutes les considérations exposées dans ce Rapport, la Commission propose à la Classe de répondre à S. Ex. le Ministre de l'Intérieur :

$1^{\circ}$ Que toutes les fabriques existantes soit dans les villes, soit aux environs, n'étant pas également susceptibles de devenir incommodes de nuire à la salubrité et de causer des inquiétudes par rapport aux accidens auxquels elles peuvent donner lieu, leur éloignement des endroits habités n'est pas non plus également nécessaire.

$2^{\circ}$ Que, pour établir les différences qui existent entre ces fabriques considérées sous le rapport des inconvéniens dont elles sont susceptibles, il convient de les diviser en trois classes.

$3^{\circ}$ Que dans la première classe on peut placer les fabriques qui, donnant naissance à des émanations incommodes et insalubres, doivent nécessairement être éloignées des habitations.

$4^{\circ}$ Que les fabriques de la seconde classe, formée de toutes celles qui, ne devenant susceptibles d'inconvéniens qu'autant que les opérations qu'on y pratique sont mal exécutées, doivent être soumises à une surveillance exacte et sévère, sans exiger quelles soient aussi éloignées que les premières. Seulement, il seroit à désirer que les grandes fabriques d'acides minéraux fussent toujours placées à l'extrémité des villes dans des quartiers peu peuplés.

3 Procédé Leblanc. 


\section{Séance du lundi 30 octobre 1809}

$5^{\circ}$ Que les fabriques de la troisième classe n'étant sujettes à aucun inconvénient n'offrent point de motifs pour qu'on ne consente pas à ce qu'elles soient placées auprès des habitations.

$6^{\circ}$ Qu'il est difficile, pour ne pas dire impossible, de déterminer les distances où il doit être permis aux fabricans de la première classe de s'établir ; mais qu'il est à propos de leur imposer d'une manière générale l'obligation de s'éloigner des lieux habités.

$7^{\circ}$ Que provisoirement on pourroit ${ }^{4}$ laisser aux autorités chargées de la police et de la surveillance des fabriques, le soin de s'assurer si les localités choisies par les fabricans ${ }^{5}$ sont à une assez grande distance des habitations ou placées de manière à ne pas porter préjudice à leurs voisins.

$8^{\circ}$ Que tout fabricant qui voudra s'établir sera tenu d'en demander la permission aux autorités compétentes et désignera en même tems le genre d'industrie qu'il se propose d'exercer.

$9^{\circ}$ Qu'avant de délivrer la permission demandée, le fabricant sera averti 6 que dans le cas où l'expérience prouveroit que les localités qu'il a choisies ne sont pas suffisamment éloignées et que les vapeurs qui s'exhalent de sa fabrique sont nuisibles sous le rapport de la salubrité ou autrement, il lui sera enjoint de porter ailleurs son établissement.

$10^{\circ}$ Que les fabricans de soude artificielle doivent être rigoureusement astreints à se placer dans des endroits inhabités et incultes, tant qu'ils n'auront pas trouvé d'autre moyen, pour se débarrasser de l'acide muriatique qu'ils séparent du muriate de soude, que de le laisser perdre dans l'atmosphère.

$11^{\circ}$ Enfin que les mesures à prendre n'auront pas un effet rétroactif pour les fabriques ou établissemens déjà en activité, pourvu toutefois qu'on ait la certitude qu'il n'y a pas eu dans leurs travaux une interruption de plus de six mois ou un an, et pourvu aussi qu'on ait la preuve que les opérations qu'on y pratique ne sont pas susceptibles de compromettre la salubrité et porter atteinte aux propriétés des voisins.

Nomenclature de divers genres de manufactures, ateliers, laboratoires, et autres établissements ayant rapport à la sûreté et à la salubrité.

$1^{\circ}$ Classe. Des fabriques qui doivent être éloignées des habitations. Amidonneries, Artificiers ${ }^{7}$, Bleu de Prusse, Boyauderies, Charbon de terre épuré, Charbon de bois

4 Dans le rapport manuscrit, ce début est remplacé par « $7^{\circ}$ Qu'il convient peut-être de ». Archives de l'Académie des sciences, Paris.

$5 \mathrm{lbid}$; ; " compris dans la $1^{\circ}$ classe $»$.

6 Dans le rapport manuscrit, ce début est remplacé par " $9^{\circ}$ Qu'il soit convenable enfin que les mêmes autorités avant d'accorder la permission de former des établissemens de l'espèce de ceux indiqués dans la $1^{\circ}$ classe se fassent connaître 11 . Archives de l'Académie des sciences, Paris.

7 Dans le rapport manuscrit, on a en plus le « Blanchiment des toiles par l'acide muriatique oxigéné 1), Archives de l'Académie des sciences. 
épuré, Chiffonniers, Colle forte, Cordes à instrumens, Cretonniers, Écarrissage, Suif brun, Ménagerie ${ }^{8}$, Minium, Fours à plâtre, Fours à chaux, Porcheries, Poudrette, Rouissage du chanvre, Sel ammoniac, Soude artificielle, Taffetas et toiles vernis, Tueries, Tourbe carbonisée, Triperie, Échaudoirs, Cuirs vernis, Fabriques de vernis, Cartonniers.

$2^{\circ}$ classe : Fabriques dont l'éloignement des habitations n'est pas aussi rigoureusement nécessaire que celui des fabriques comprises dans la 1 ère classe, mais qui doivent être assujetties à une surveillance. Blanc de céruse ${ }^{9}$, Chandeliers, Corroyeurs, Couvertures, Dépôts de cuirs verts, Distillerie d'eau-de-vie, Eau forte, Acide sulfurique, Acide muriatique et nitrique, Fonderie de métaux, Affinage des métaux au fourneau à manche, Suif en branche, Noir d'ivoire, Noir de fumée, Plomberies, Plomb de chasse, Salles de dissection, Fabriques de tabac, Taffetas cirés, Vacheries, Teinturiers, Hongroyeurs, Mégisseurs, Les pompes à feu, Blanchiment des toiles par l'acide muriatique oxigéné.

$3^{\circ}$ classe : Fabriques qui peuvent rester auprès des habitations. Alun, Vitriols, Boutons, Brasserie, Ciriers, Colle de parchemin etd'amidon, Cornes transparentes, Dorures sur métaux, Caractères d'imprimerie, Papiers peints, Savonneries, etc.

Signé à La minute: Chaptal, Fourcroy, Vauquelin, Guyton-Morveau, Deyeux Rapporteur.

La Classe approuve le Rapport et en adopte les conclusions. ॥

8 Dans le rapport manuscrit, on a ici en plus la « Mégisserie » (placée en définitive en $2^{\circ}$ classe). 9 Dans le rapport manuscrit, on a ici en plus les « Cartonniers ». 


\title{
Décret impérial relatif aux établissements et ateliers qui répandent une odeur insalubre ou incommode, au Palais de Fontainebleau,
} le 15 octobre 1810

\author{
(retranscription)
}

\section{Préfecture de Police}

Préfecture de Police. Décret impérial relatif aux établissements et ateliers qui répandent une odeur insalubre ou incommode, au Palais de Fontainebleau, le 15 octobre $1810^{\prime}$.

Napoléon, Empereur des Français, Roi d'Italie, Protecteur de la Confédération du Rhin, Médiateur de la Confédération suisse,

Sur le rapport de notre Ministre de l'Intérieur ;

Vu les plaintes portées par différents particuliers contre les manufactures et ateliers dont l'exploitation donne lieu à des exhalaisons insalubres ou incommodes:

Le rapport fait sur ces établissements par la section de Chimie de la Classe des Sciences physiques et mathématiques de I'Institut; Notre Conseil d'État entendu, Nous avons décrété et décrétons ce qui suit :

Article Premier.

A compter de la publication du présent décret, les manufactures et ateliers qui répandent une odeur insalubre ou incommode ne pourront être formés sans une permission de l'autorité administrative ; ces établissements seront divisés en trois classes :

La première classe comprendra ceux qui doiventêtre éloignés des habitations particulières ;

La seconde, les manufactures et ateliers dont l'éloignement des habitations n'est pas rigoureusement nécessaire, mais dont il importe néanmoins de ne permettre la formation qu'après avoir acquis la certitude que les opérations qu'on y pratique sont exécutées de manière à ne pas incommoder les propriétaires du voisinage, ni à leur causer des dommages ;

Dans la troisième classe seront placés les établissements qui peuvent rester sans inconvénient auprès des habitations, mais doivent rester soumis à la surveillance de la Police.

1 Préfecture de Police, Recueil des décrets et ordonnances du Préfet de Police, Paris, 1839, I, pp. 93-95. 
Art. 2. - La permission nécessaire pour la formation des manufactures et ateliers compris dans la première classe sera accordée avec les formalités ciaprès, par un décret rendu en notre Conseil d'État.

Celle qu'exigera la mise en activité des établissements compris dans la seconde classe le sera par les Préfets, sur l'avis des Sous-Préfets.

Les permissions pour l'exploitation des établissements classés dans la troisième classe seront délivrés par les Sous-Préfets, qui prendront préalablement l'avis des Maires.

Art. 3. - La permission, pour les manufactures et fabriques de première classe, ne sera accordée qu'avec les formalités suivantes:

La demande en autorisation sera présentée au préfet et affichée par son ordre dans toutes les communes, à 5 kilomètres de rayon.

Dans ce délai, tout particulier sera admis à présenter ses moyens d'opposition. Les Maires des communes auront la même faculté.

Art. 4. - S'il y a des oppositions le Conseil de Préfecture donnera son avis, sauf la décision du Conseil d'État.

Art. 5. - S'il n'y a pas d'opposition, la permission sera accordée, s'il y a lieu, sur l'avis du Préfet et le rapport de notre Ministre de l'Intérieur.

Art. 6. - S'il s'agit de fabriques de soude, ou si la fabrique doit être établie dans la ligne des douanes, notre Directeur général des Douanes sera consulté.

Art. 7. - L'autorisation de former des manufactures et ateliers compris dans la seconde classe ne sera accordée qu'après que les formalités suivantes auront été remplies :

L'entrepreneur adressera d'abord sa demande au Sous-Préfet de son arrondissement, qui la transmettra au maire de la commune dans laquelle on projette de former l'établissement, en le chargeant de procéder à des informations de " commodo " et " incommodo ». Ces informations terminées, le Sous-Préfet prendra, sur le tout, un arrêté qu'il transmettra au Préfet. Celui-ci statuera, sauf le recours à notre conseil d'État par toutes parties intéressées.

S'il y a opposition, il y sera statué par le Conseil de Préfecture, sauf le recours au Conseil d'État.

Art. 8. - Les manufactures et ateliers ou établissements portés dans la troisième classe, ne pourront se former que sur la permission du Préfet de Police, à Paris, et sur celle du Maire, dans les autres villes.

S'il s'élève des réclamations contre la décision prise par le Préfet de Police ou les Maires, sur une demande en formation de manufacture ou d'atelier compris dans la troisième classe, elles seront jugées au Conseil de préfecture.

Art. 9. - L'autorité locale indiquera le lieu ou les manufactures et ateliers compris dans la première classe pourront s'établir, et exprimera sa distance des habitations particulières. Tout individu qui ferait des constructions dans le voisinage de ces manufactures et ateliers, après que la formation en aura été permise, ne sera plus admis à en solliciter l'éloignement.

Art. 10. - La division en trois classes des établissements qui répandent une odeur insalubre ou incommode, aura lieu conformément au tableau annexé au présent décret. Elle servira de règle toutes les fois qu'il sera question de se prononcer sur des demandes en formation de ces établissements.

Art. 11. - Les dispositions du présent décret n'auront point d'effet rétroactif ; en conséquence, tous les établissements qui sont aujourd'hui en activité continueront à être exploités librement, sauf les dommages dont pourront être passibles les entrepreneurs de ceux qui préjudicient aux propriétés de leurs voisins ; les dommages seront arbitrés par les tribunaux. 


\section{Décret impérial relatif aux établissements insalubres ou incommodes}

Art. 12. - Toutefois, en cas de graves inconvénients pour la salubrité publique, la culture, ou l'intérêt général, les fabriques et ateliers de première classe qui les causent pourront être supprimés, en vertu d'un décret rendu en notre Conseil d'État, après avoir entendu la police locale, pris l'avis des Préfets, reçu la défense des manufacturiers ou fabricants.

Art. 13. - Les établissements maintenus par l'article 11 cesseront de jouir de cet avantage, dès qu'ils seront transférés dans un autre emplacement, ou qu'il y aura une interruption de six mois dans leurs travaux. Dans l'un et l'autre cas, ils rentreront dans la catégorie des établissements à former, et ils ne pourront être remis en activité qu'après avoir obtenu, s'il y a lieu, une nouvelle permission.

Art. 14. - Nos Ministres de l'Intérieur et de la Police générale sont chargés, chacun en ce qui le concerne, de l'exécution du présent décret, qui sera inséré au « Bulletin des Lois ».

Signé Napoléon

Pour l'Empereur, le Ministre Secrétaire d'État. Signé, H. B. Duc de Bassano

Nomenclature des Manufactures, Établissements et Ateliers répandant une odeur insalubre ou incommode, dont la formation ne pourra avoir lieu sans une permission de l'Autorité administrative.

Éłablissemens et ateliers qui ne pourront plus être formés dans le voisinage des habitations particulières, et pour la création desquels il sera nécessaire de se pourvoir de l'autorisation du Ministre de l'intérieur.

$\begin{array}{ll}\text { Amidoniers. } & \text { Fours à chaux. } \\ \text { Artificiers. } & \text { Porcheries. } \\ \text { Bleu de Prusse. } & \text { Poudrette. } \\ \text { Boyaudiers. } & \text { Rouissage du chanvre. } \\ \text { Charbon de terre épuré. } & \text { Sel ammoniac. } \\ \text { Charbon de bois épuré. } & \text { Soude artificielle. } \\ \text { Chiffonniers. } & \text { Taffetas et toiles vernis. } \\ \text { Colle-forte. } & \text { Tueries. } \\ \text { Cordes à instrumens. } & \text { Tourbe carbonisée. } \\ \text { Cretonniers. } & \text { Triperies. } \\ \text { Écarrissage. } & \text { Échaudoirs. } \\ \text { Eau-forte, acide sulfurique, etc. } & \text { Cuirs vernis. } \\ \text { Suif brun. } & \text { Cartonniers. } \\ \text { Ménagerie. } & \text { Fabriques de vernis. } \\ \text { Minium. } & \text { Fabriques d'huile de pied ou de come de bœuf. }\end{array}$

Four \& à plâtre. 
Établissemens et ateliers dont l'éloignement des habitations n'est pas rigoureusement nécessaire, mais dont il importe néanmoins de ne permettre la formation qu' après avoir acquis la certitude que les opérations qu' on y pratique sont exécutées de manière à ne pas incommoder les Propriétaires du voisinage, ni à leur causer des dommages. Pour former ces établissemens, l'autorisation du Préfet sera nécessaire.

Blanc de céruse.

Chandeliers.

Corroyeurs.

Couverturiers.

Dépôts de cuirs verts.

Distilleries d'eau-de-vie.

Fonderies de métaux.

Affinage des métaux au foumeau à manche.

Teinturiers

Hongroyeurs.

Mégissiers.

Pompes-à-feu.
Suif en branche.

Noir d'ivoire.

Noir de fumée.

Plomberies.

Plomb de chasse.

Salles de dissection.

Fabriques de tabac.

Taffetas cirés.

Vacheries.

Blanchiment des toiles par l'acide muriatique oxigéné. Les filatures de soie.

Établissemens et ateliers qui peuvent rester sans inconvénient auprès des habitations particulières, et pour la formation desquels il sera nécessaire de se munir d'une permission du Sous-Préfet.

Alun.

Boutons.

Brasseries.

Ciriers.

Colle de parchemin et d'amidon.

Cornes transparentes.
Caractères d'imprimerie.

Doreurs sur métaux.

Papiers peints.

Savonneries, etc.

Vitriols. 


\title{
Ordonnance concernant les Manufactures et Ateliers qui répandent une odeur insalubre ou incommode approuvée par S. Exc. le Ministre de l'intérieur, le 17 novembre 1810
}

\author{
(retranscription)
}

\section{Ministère de l'Intérieur}

Ordonnance concernant les Manufactures et Ateliers qui répandent une odeur insalubre ou incommode approuvée par S. Exc. le Ministre de l'intérieur, le 17 novembre 1810 '.

«Paris, le 5 Novembre 1810.

Nous, Étienne-Denis Pasquier, Chevalier de la Légion d'honneur, Baron de l'Empire, Conseiller d'État, chargé du $4^{\mathrm{e}}$ arrondissement de la Police générale, Préfet de Police du département de la Seine et des Communes de Saint-Cloud, Sèvres et Meudon du département de Seine et Oise, etc.

Vû les articles 2 et 23 de l'Arrêté du Gouvernement du 12 messidor an 8, et l'article ler de celui du 3 brumaire an 9 ;

Ordonnons ce qui suit :

Article $1^{\mathrm{er}}$. Le Décret impérial du 15 octobre 1810, relatif aux Manufactures et Ateliers qui répandent une odeur insalubre ou incommode, ensemble le Tableau y annexé, seront imprimés, publiés et affichés, avec la présente Ordonnance, dans le ressort de la Préfecture de Police.

II. Les demandes en autorisation pour former des Manufactures ou Ateliers compris dans la première classe du tableau annexé au Décret précité, nous seront adressées pour être par nous procédé conformément aux articles 3, 4, 5 , 6 et 9 du Décret.

III. Les demandes en autorisation, pour former des Manufactures ou Ateliers compris dans la deuxième classe seront adressées, savoir :

1. Pour Paris, au Préfet de Police :

2. ${ }^{\circ}$ Pour les Communes rurales du Département de la Seine, aux Sous-Préfets de S.-Denis et de Sceaux ;

3. Et pour les Communes de Saint-Cloud, Sèvres et Meudon, aux Maires de ces Communes.

1 Préfecture de Police, Recueil des décrets et ordonnances du Préfet de Police, Paris, 1832, I, p. 136. 
II sera par nous statué sur ces demandes, conformément à l'article VII du Décret.

IV. Les demandes en autorisation pour former des Manufactures ou Ateliers compris en la $3^{\text {ème }}$ classe, nous seront adressées pour être par nous statué, conformément à l'article VIII du Décret.

V. Les Propriétaires ou Entrepreneurs énonceront dans leurs demandes, la nature des matières qu'ils se proposent de préparer dans leurs Manufactures ou Ateliers et des travaux qui devront être exécutés; ils déposeront en même-tems, un plan figuré des lieux et des constructions projettées.

VI. Indépendamment des formalités prescrites par le Décret, il sera procédé, par le Conseil de Salubrité établi près la Préfecture de Police assisté de l'Architecte-Commissaire de la petite Voirie, à la visite des lieux, à l'effet de s'assûrer si l'établissement projetté ne peut nuire à la salubrité, ni faire craindre un incendie.

VII. Les Propriétaires d'une Manufacture ou Atelier aujourd'hui en activité dans le ressort de la Préfecture de Police, seront tenus d'en faire la déclaration avant le premier janvier prochain ; savoir :

$1{ }^{\circ}$ Dans Paris, à la Préfecture de Police ;

$2^{\circ}$ Dans les Communes rurales du Département de la Seine, aux Sous-Préfets de S.-Denis et de Sceaux;

$3^{\circ}$ Dans les Communes de S.-Cloud, Sèvres et Meudon, aux Maires de ces Communes.

VIII. Les Sous-Préfets des arrondissemens de S.-Denis et de Sceaux et les Maires des Communes de S.-Cloud, Sèvres et Meudon, enverront à la Préfecture de Police, l'état des déclarations qu'ils auront reçues.

IX. La présente Ordonnance sera soumise à l'approbation de S. Ex. le Ministre de l'interieur.

X. Les Sous-Préfets des arrondissemens de S.-Denis et de Sceaux, les Maires des Communes rurales du ressort de la Préfecture de Police, les Commissaires de Police, l'Inspecteur-général du $4^{\text {ème }}$ arrondissement de la Police générale de l'Empire, les Officiers de Paix, l'Architecte-Commissaire de la petite Voirie, les Commissaires des Halles et Marchés, I'Inspecteur-général de la Salubrité et les autres Préposés de la Préfecture de Police, sont chargés de tenir la main à son exécution.

Le Conseiller d'État, Préfet, Baron de l'Empire, Signé, Pasquier.

Par le Conseiller d'État, Préfet, Le Secrétaire-général, Chevalier de l'Empire,

Signé, Piis $॥$. 


\title{
Instruction Relative aux Manufactures De Bleu de Prusse
}

\section{(retranscription et fac-similé)}

\author{
Préfecture de Police \\ Conseil de salubrité
}

Préfecture de Police.

CONSEIL DE SALUBRITE

Paris, le 21 avril 1812.

Instruction Relative aux Manufactures De Bleu de Prusse?.

La fabrication du Bleu de Prusse ${ }^{2}$ exige deux opérations pendant lesquelles il se dégage une assez grande quantité de vapeurs nuisibles ou au moins bien incommodes.

La première est la calcination des matières animales mélangées avec la potasse.

La seconde est la précipitation du sulfate de fer ${ }^{3}$ et du sulfate d'alumine ${ }^{4}$ par la lessive du prussiate de potasse ${ }^{5}$ procédé que les fabricants appellent fixation.

Dans la première de ces opérations il se dégage de l'huile empyreumatique', du carbonate d'ammoniaque et du gaz hydrogène carboné.

Dans la seconde, il se dégage du gaz hydrogène sulfuré.

Il est important, il est facile de coërcer ${ }^{7}$ ces gaz et ces vapeurs par les moyens suivants.

\footnotetext{
1 Instruction du 18 mai 1812 du Préfet de Police, soumise à l'approbation du Ministre des manufactures et du commerce. Elle est révisée par le Comité des arts et manufactures et publiée le 17 septembre 1813.

2 Appelé aussi bleu de Berlin, bleu Raymond ou bleu Marie-Louise. Ce prussiate de potasse est le premier colorant de synthèse découvert à Berlin en 1710 par le pharmacien Dippel et le coloriste Diesbach dont la préparation ne cesse de s'améliorer à la fin du XVIIIle siècle : André Guillerme, La naissance de l'industrie à Paris (1780-1830), Seyssel, Champ Vallon, 2007, pp. 163-171 ; Sabine Barles, L'invention des déchets urbains. France : 1790-1970, Seyssel, Champ Vallon, 2005, pp. 143-144. Napoléon souhaite développer sa production en complément de la consommation d'indigo qui atteint 18 millions de francs en 1806 (dont 12 d'importation). Le chimiste Raymond à Lyon remporte le prix de la Société d'encouragement pour l'industrie nationale en 1811 pour l'application du colorant au mordançage des textiles.

3 Appelé aussi couperose, provenant d'Alsace, du Beauvaisis ou fabriquée par les chimistes en dissolvant du fer dans de l'acide sulfurique dilué.

4 Nom chimique de l'alun soit naturel, provenant des mines de Toffa (Italie) ou de Liège ou de l'Aveyron, soit synthétique par torréfaction d'argile et de sel marin.

5 C'est pour l'essentiel du cyanure de potasse, formule chimique : $\mathrm{KCN}$.

6 Produit chimique obtenu soit par la putréfaction des matières organiques azotées (chiffons, cuirs, eaux mères de salpêtre, chaux) distillées, soit par la décomposition des déchets alimentaires putréfiés par l'acide chlorhydrique et le gypse ; épuré, il donne l'huile de Dippel alors recommandée contre l'épilepsie.

7 Concentrer et détruire dans une cornue.
} 


\section{Dréfecture de Lolicew.}

CONSEIL DE SALUBRITÉ.

Qaric, lo 21 anil 1812.

Instruetion Retative aux Manufactures

$$
\text { De Heu De frusse. }
$$

$8^{i 5}$

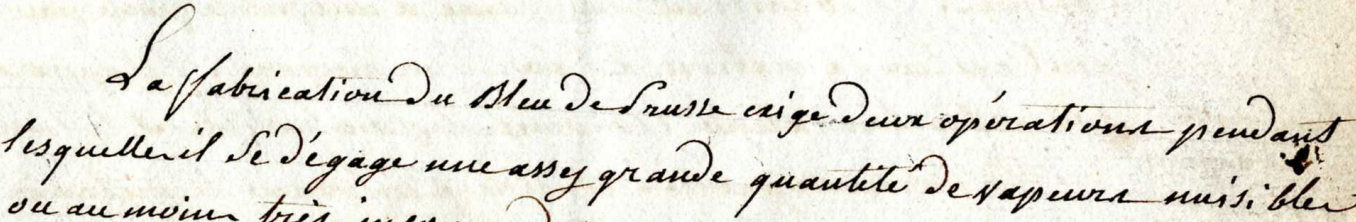

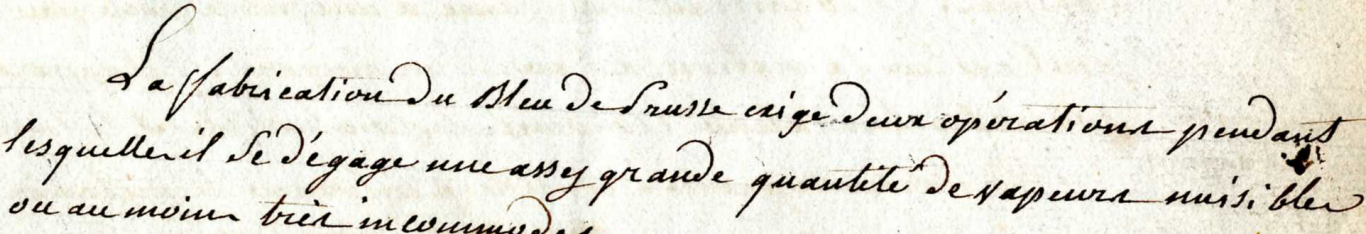
1 , tre avee la polaster. Aa prémiere est ta ealcinatiere?

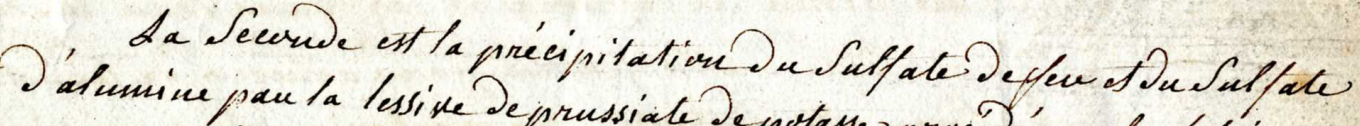

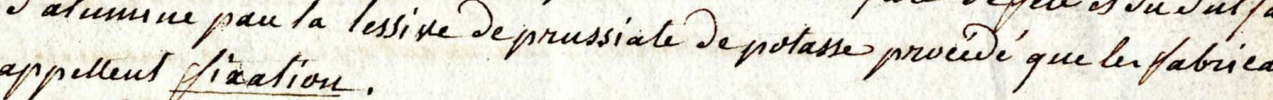
Damba presuce Lupigrenmatique, ? ear Gormé.

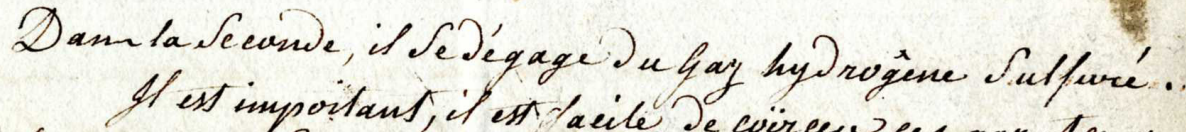
pacelesuryen Sirane.

$$
\text { Calcinalion. }
$$

Le fabricant De Dlen en russe qui evmait bien Sou art, Sait quil

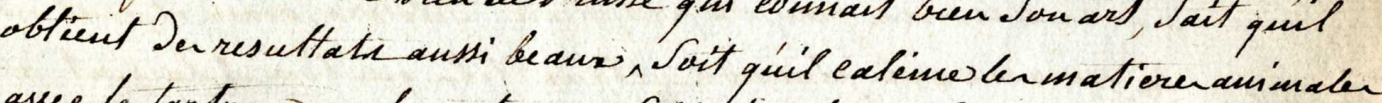

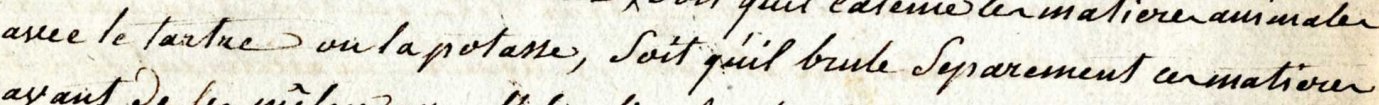

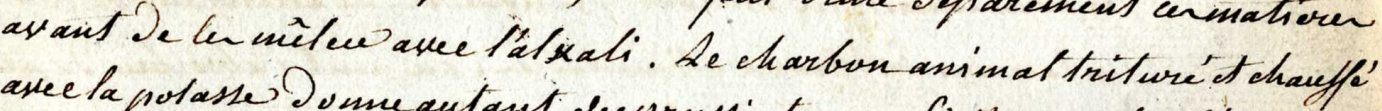

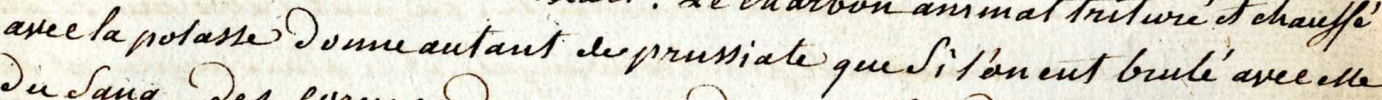
Dis Sang, Des corser, Der peanx, Des muscles; Des poild of auber.

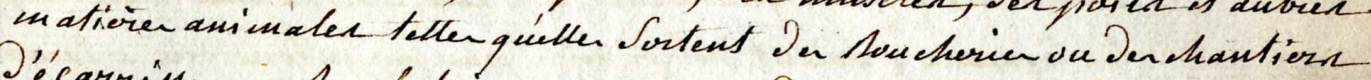
(7) Décarrissage. Aerfabrieanis peusent Done némplorgen Dam lewn atelièn 


\section{Calcination}

Le fabricant de Bleu de Prusse qui connaît bien son art, sait qu'il obtient des résultats aussi beaux : soit qu'il calcine les matières animales avec le tartre ou la potasse, soit qu'il brule séparément ces matières avant de les mêler à l'alkali ${ }^{8}$. Le charbon animal ${ }^{9}$ trituré et chauffé avec la potasse donne autant de prussiate que si l'on eut brulé avec elle du sang, des cornes, des peaux, des muscles, des poils et autres matières animales telles qu'elles sortent des boucheries ou des chantiers d'écarrissage. Les fabricants peuvent donc n'employer dans leurs ateliers que du charbon animal fait en pleine campagne et dans des lieux isolés ou si pour des motifs d'économie, ils ne veulent faire qu'une seule calcination, ils peuvent suivre l'un des trois procédés suivant pour calciner sans que les vapeurs soient nuisibles en dehors.

Le premier consiste à bruler les matières dans un petit four à réverbère ${ }^{10}$ dont la température est fort élevée avant qu'on y projette le mélange. Les frères Gohin "', fabricants de couleurs rue du faubourg St Martin [à Paris] se servent avec succès de ce procédé.

Le second à couvrir le creuset de calcination avec un chapiteau de tôle garni de terre et terminé par un tuyau de poële qui porte la fumée dans un corps de cheminée s'élevant à la hauteur de celle des maisons voisines. Ce chapiteau ou Dôme a une petite porte par laquelle on peut agiter la matière à l'aide d'un ringard. Cet appareil (voyez figure) concentre la chaleur, économise le combustible et la terre. II faut mettre le feu aux vapeurs qui se dégagent du creuset, aussitôt qu'elles sont assez chaudes pour pouvoir s'enflammer.

Le troisième procédé consiste a enfermer le mélange dans un appareil distillatoire, c'est-à-dire dans un tuyau de fonte ou de forte tôle qui traverse un pot et auquel est adapté un récipient pneumatochimique qui après avoir fait passer les vapeurs et gaz dans l'eau, les conduit soit dans un foyer particulier, soit dans le foyer même du fourneau pour achever de les détruire ${ }^{12}$. On trouve dans cette méthode deux avantages. Le premier de n'avoir aucune odeur désagréable, le second de pouvoir recueillir le carbonate d'ammoniaque qui a une valeur dans le commerce ${ }^{13}$ (voyez fig. 2).

\footnotetext{
8 Base. Ici potasse $(\mathrm{KOH})$.

9 Appelé aussi noir d'os, mélange de phosphate et de carbonate de chaux, d'oxyde de calcium et de carbone dont la principale propriété est d'absorber les colorants et de purifier. Il est obtenu en torréfiant des os dans des marmites en terre. D'abord reconnu par le chimiste Thouret à La Monnaie, vers 1750, pour séparer l'argent de l'or des cendres d'orfèvre, puis, vers 1785, pour filtrer l'eau de boisson, vers 1815 pour blanchir la mélasse et enfin, vers 1825, servir d'engrais maraîcher. 10 Four chauffé au charbon de bois, doté d'une paroi cylindrique qui concentre la chaleur et augmente ainsi la température.

11 Chimistes installés depuis 1803. Ils fabriquent aussi des vernis à La Chapelle pour leur importante hongroierie (une centaine d'ouvriers) : ils fournissent ainsi de magnifiques harnais bleus à la Cavalerie impériale. Rue Jussienne, ils fabriquent des bottes et y emploient une cinquantaine de corroyeurs. 12 Foyer fumivore mis au point par d'Arcet dans les années 1810.

13 Pour la verrerie.
} 


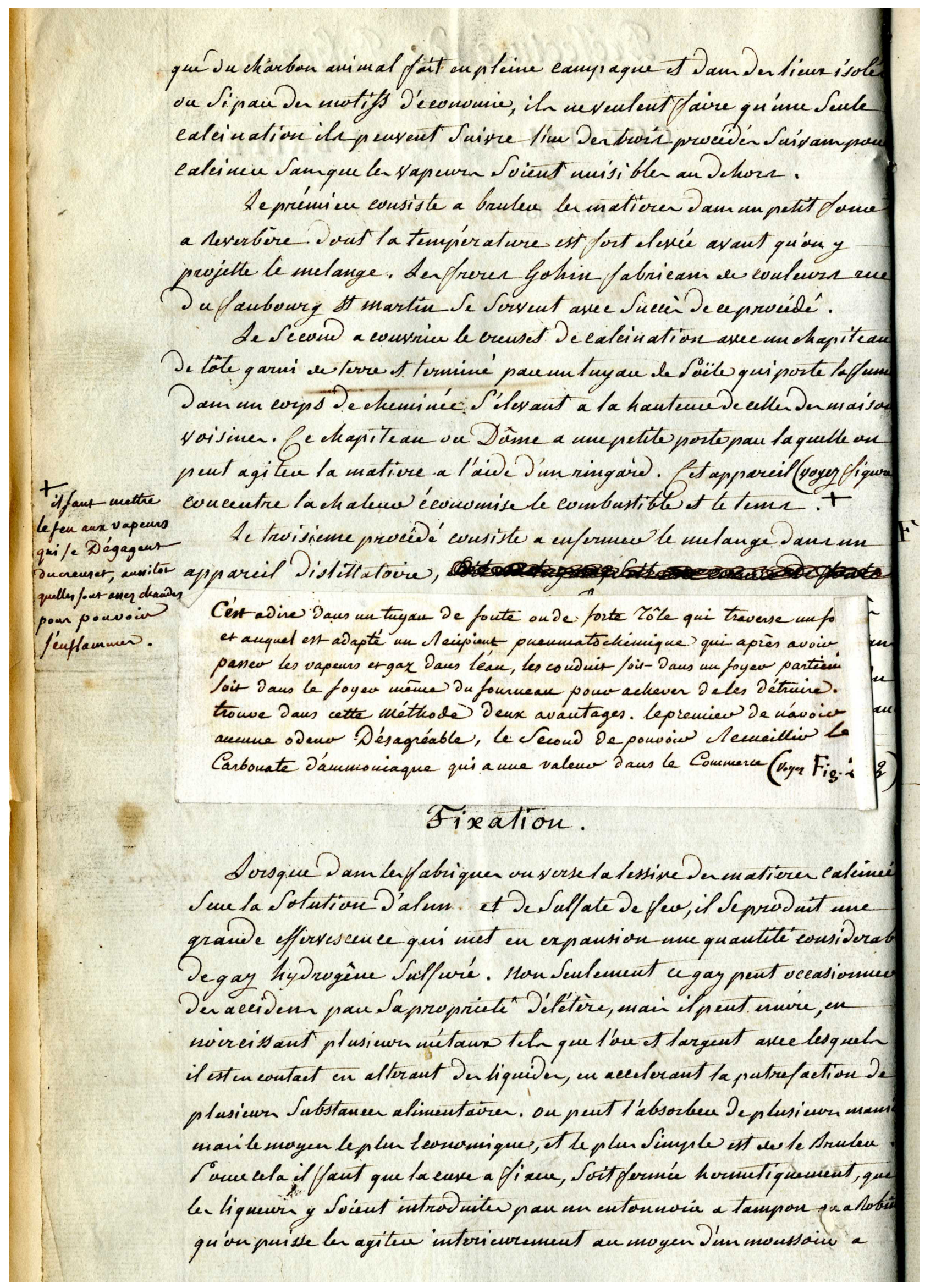


Fixation

Lorsque dans les fabriques on verse la lessive des matières calcinées sur la solution d'alun et le sulfate de fer, il se produit une grande effervescence qui met en expansion une quantité considérable de gaz hydrogène sulfuré. Non seulement ce gaz peut occasionner des accidents par sa propriété délétère, mais il peut nuire, en noircissant plusieurs métaux tels que l'or et l'argent avec lesquels il est en contact, en altérant des liquides, en accélérant la putréfaction de plusieurs substances alimentaires. On peut l'absorber de plusieurs manières, mais le moyen le plus économique et le plus simple est de le brûler.

Pour cela, il faut que la cuve à fixer, soit fermée hermétiquement, que les liqueurs y soient introduites par un entonnoir à tampon ou à robinet, qu'on puisse les agiter intérieurement au moyen d'un moussoir à manivelle, qu'un tuyau adapté au fond supérieur de la cuve conduise le gaz dans un petit baril aux deux tiers plein d'eau et muni d'un tube de sûreté, que de ce baril parte un autre tuyau qui porte le gaz dans un foyer allumé avant la fixation.

Cet appareil a déjà parfaitement réussi dans plusieurs stations notamment dans celui de Mr Bronstrom ${ }^{14}$, petite rue St Nicolas, faubourg St Martin (voyez fig. 3).

Les fabricants de Bleu de Prusse qui voudront ajouter à ces instructions des renseignements plus étendus trouveront des conseils très utiles dans la chimie appliquée aux arts par Mr le comte Chaptal' ${ }^{15}$, tome 4, pages 260 jusqu'à 271, dans la statique chimique de Mr le comte Berthollet ${ }^{16}$, tome 2, page 243, dans un mémoire de Mr Proust ${ }^{17}$ inséré dans les Annales de chimie tome 22.

Lu et approuvé au Conseil de Salubrité

Le 21 avril 1812

Signé : Pariset, Parmentier, Dupuytren, C.L. Cadet de Gassicourt, Thouret, Deyeux, Huzard.

\footnotetext{
14 Installé depuis 1803 au n 37, ce Suédois ami de Bergmann, fabrique alors du bleu en grande quantité. Le voisinage se plaint beaucoup. "Aux odeurs de corne brûlée émanant de la synthèse du prussiate s'ajoutent celles d'œuf pourri ou de latrines, dégagement d'acide sulfureux provenant des cuves à ciel ouvert » [Rapport n ${ }^{\circ} 4$ du Conseil de Salubrité du 7 juin 1806]. Vauquelin, Thénard, chimistes, Deyeux et Cadet, pharmaciens, qui ont visité les lieux à la demande expresse du préfet de Police, préconisent de couvrir les chaudières par une hotte en céramique et de rehausser la cheminée principale de trois ou quatre mètres au-dessus des maisons voisines. La bonne volonté de Brostrom assure le succès : les plaintes cessent et le conseil demande au préfet d'obliger tous les fabricants de bleu de Prusse à user du même équipement.

15 Jean-Antoine Chaptal, Chimie appliquée aux arts, Paris, 1807, 3 vol.

16 Claude-Louis Berthollet, Statique chimique, Paris, 1789, 2 vol.

17 Joseph-Louis Proust, « Le bleu de Prusse ॥, Annales de chimie, 22, 1804, pp. 27-38.
} 


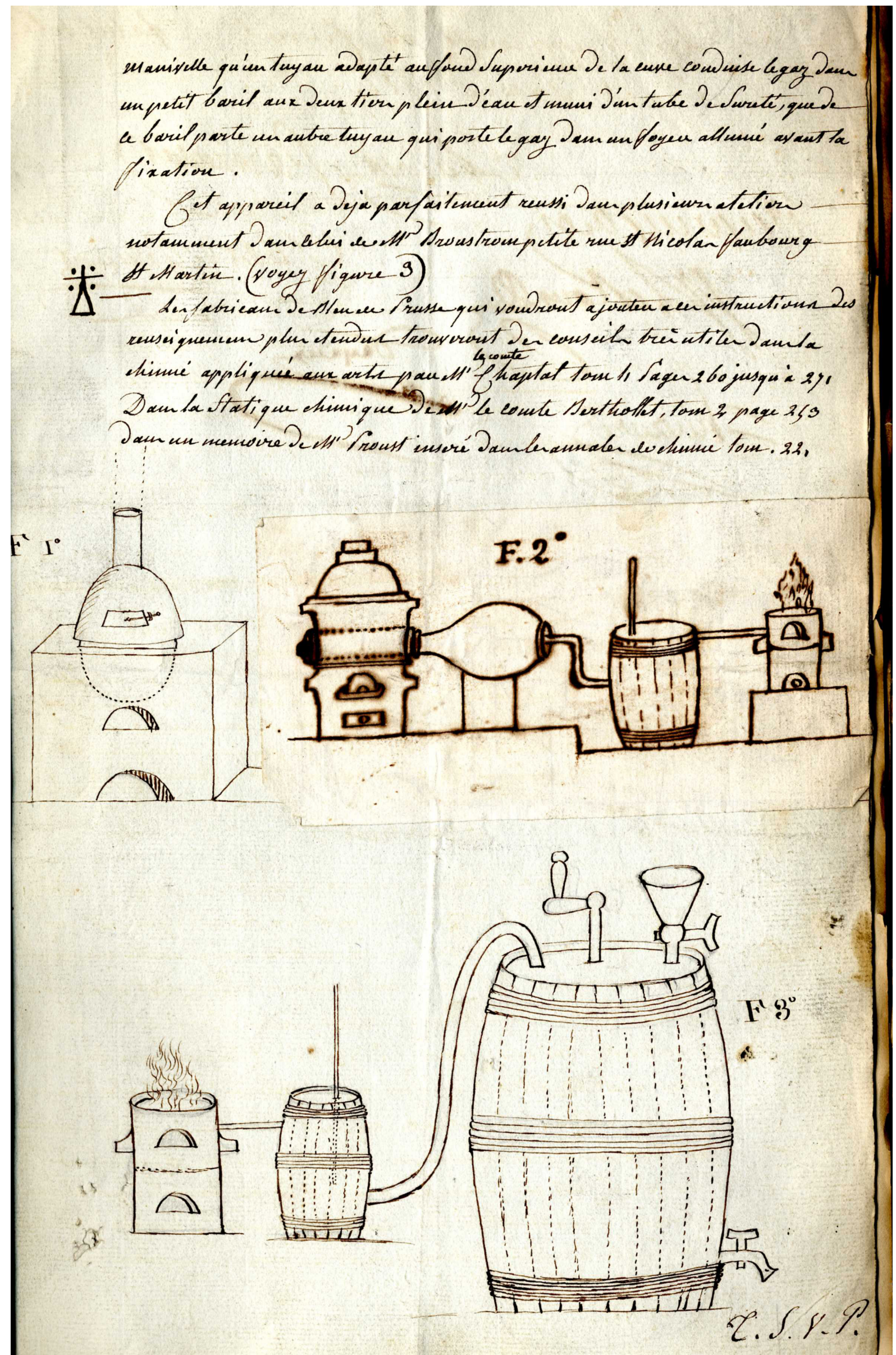




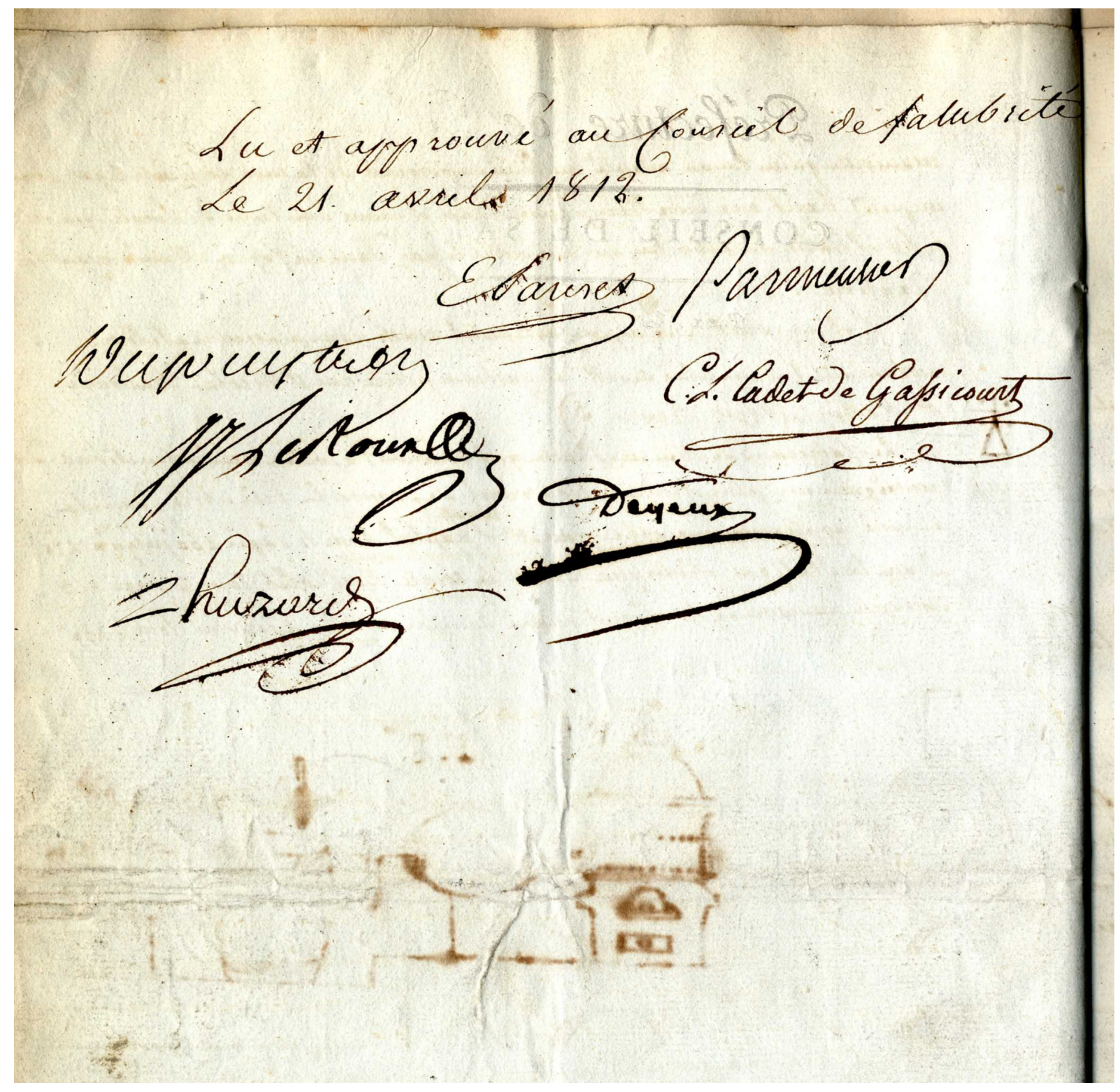

